\title{
The Dirichlet problem with BMO boundary data and almost-real coefficients
}

\author{
Ariel Barton
}

\begin{abstract}
It is known that a function, harmonic in a Lipschitz domain, is the Poisson extension of a BMO function if and only if its gradient satisfies a Carleson-measure condition. We show that the same is true of functions that satisfy elliptic equations in two-dimensional Lipschitz domains, provided the coefficients are independent of one coordinate and have small imaginary part.
\end{abstract}

\section{Introduction}

Consider the Dirichlet problem

$$
\left\{\begin{aligned}
\operatorname{div} A \nabla u=0 & & \text { in } V, \\
u=f & & \text { on } \partial V,
\end{aligned}\right.
$$

where $A$ is a uniformly elliptic matrix, that is, where there exist constants $\Lambda>$ $\lambda>0$ such that

$$
\lambda|\eta|^{2} \leq \operatorname{Re} \bar{\eta} \cdot A(X) \eta, \quad|\xi \cdot A(X) \eta| \leq \Lambda|\xi||\eta|
$$

for all $X \in \mathbb{R}^{2}$ and all $\eta, \xi \in \mathbb{C}^{2}$.

In this paper we study the Dirichlet problem (1.1) in the case where the boundary data $f$ lies in the space BMO of functions of bounded mean oscillation, where the domain $V$ is a two-dimensional Lipschitz domain, and where the coefficients $A$ are complex but have small imaginary part, and are $t$-independent in the sense that

$$
A(x, t)=A(x, s) \text { for all } \quad x, s, t \in \mathbb{R} .
$$

Mathematics Subject Classification (2010): Primary 35J25; Secondary 31A25.

Keywords: Elliptic equations, Dirichlet problem, Lipschitz domains, Carleson measures, bounded mean oscillation, layer potentials. 
This paper is an extension of the author's earlier work [8], which instead considered boundary data $f$ in the Lebesgue space $L^{p}(\partial V)$. The study of elliptic boundaryvalue problems has a long and rich history; we refer the reader to Section 1.1 of [8] for a more detailed history of the Dirichlet problem with boundary data in $L^{p}(\partial V)$.

In this paper we will prove the following theorem.

Theorem 1.4. Suppose that $A$ is t-independent and elliptic, and that $V \subset \mathbb{R}^{2}$ is a two-dimensional Lipschitz domain with connected boundary.

Then there exist constants $\varepsilon>0$ and $C>0$, depending only on the constants $\lambda$ and $\Lambda$ in the bound (1.2) and the Lipschitz character of $V$, such that if $\|\operatorname{Im} A\|_{L^{\infty}}<\varepsilon$, then the following holds.

For all $f \in \mathrm{BMO}(\partial V)$ there exists a function $u$ that satisfies the conditions (1.1) and such that, for all $X_{0} \in \partial V$ and all $r>0$,

$$
\frac{1}{\sigma\left(\partial V \cap B\left(X_{0}, r\right)\right)} \int_{V \cap B\left(X_{0}, r\right)}|\nabla u(X)|^{2} \operatorname{dist}(X, \partial V) d X \leq C\|f\|_{\mathrm{BMO}}^{2} .
$$

Furthermore, $u$ is unique among solutions to the problem (1.1) for which the lefthand side of (1.5) is bounded uniformly in $X_{0}$ and $r$.

Conversely, if $\operatorname{div} A \nabla u=0$ in $V$ and if

$$
\sup _{X_{0} \in \partial V} \sup _{r>0} \frac{1}{\sigma\left(\partial V \cap B\left(X_{0}, r\right)\right)} \int_{V \cap B\left(X_{0}, r\right)}|\nabla u(X)|^{2} \operatorname{dist}(X, \partial V) d X \leq \widetilde{C}^{2},
$$

then $f=\left.u\right|_{\partial V}$ exists and lies in $\operatorname{BMO}(\partial V)$, with $\|f\|_{\mathrm{BMO}} \leq C \widetilde{C}$.

We will also establish the following maximum principle for such coefficients.

Theorem 1.7. Let $A$ and $V$ be as in Theorem 1.4. Then there is a constant $\varepsilon>0$ such that, if $\|\operatorname{Im} A\|_{L^{\infty}}<\varepsilon$, then for each $f \in L^{\infty}(\partial V)$ there exists a unique function $u$ that solves the problem (1.1) and such that $u \in L^{\infty}(V)$. Furthermore, there is a constant $C$ such that

$$
\|u\|_{L^{\infty}(V)} \leq C\|f\|_{L^{\infty}(\partial V)} .
$$

In Theorems 1.4 and 1.7 the coefficients $A$ are taken to be independent of the second coordinate. We would like to emphasize that the domain $V$ in these theorems may be bounded, and thus the distinguished direction (in which $A$ is constant) need not be transverse to the boundary $\partial V$. This is the setting of the papers [24], [34], [8], but is distinct from the setting of many other papers (such as [23], [26], [6], [2], [3], [1], [33], [18], [20], [21], and [5]), where the coefficients $A$ are taken to be constant specifically in a direction transverse to the boundary.

We now review the history of the BMO-Dirichlet problem. In [15], Fefferman proved that the Poisson extension of a BMO function in the upper half-space satisfies the bound (1.5). In [13], Fabes, Johnson and Neri proved that a function $u$ harmonic in the upper half-space that satisfies the condition (1.6) was necessarily the Poisson extension of a BMO function. Thus, Theorem 1.4 is valid for harmonic 
functions (that is, for $A \equiv I$ ) in the upper half-space. Later in [14], Fabes and Neri extended these results to harmonic functions in arbitrary starlike Lipschitz domains.

Many of the known results concerning the Dirichlet problem (1.1) for more general coefficients involve boundary data in Lebesgue spaces $L^{p}(\partial V)$, rather than in $\operatorname{BMO}(\partial V)$. We say that the $L^{p}$-Dirichlet problem is well-posed if, for each $f \in L^{p}(\partial V)$, there exists a unique function $u$ that solves the problem (1.1) and such that the nontangential maximal function $N u$ lies in $L^{p}(\partial V)$, where

$$
N u(X)=\sup \{|u(Y)|:|X-Y|<(1+a) \operatorname{dist}(Y, \partial V)\}
$$

for some constant $a>0$. The following result suggests a connection between the $L^{p}$-Dirichlet problem and the BMO-Dirichlet problem of Theorem 1.4. Suppose that $V$ is a bounded Lipschitz domain (of arbitrary dimension), that $X_{0} \in V$, and that the $L^{p}$-Dirichlet problem is well-posed in $V$ for some real coefficient matrix $A$. Then the inequalities

$$
\frac{1}{C} \int_{\partial V} N u^{2} d \sigma \leq \int_{V}|\nabla u(X)|^{2} \operatorname{dist}(X, \partial V) d X \leq C \int_{\partial V} N u^{2} d \sigma
$$

are valid for any $u$ that satisfies $\operatorname{div} A \nabla u=0$ in $V$ and $u\left(X_{0}\right)=0$, where $C$ depends on $A, V$ and $X_{0}$. This was established by Dahlberg in [9] (for harmonic functions) and by Dahlberg, Jerison and Kenig in [10] (for more general real coefficients).

In [24], Kenig, Koch, Pipher and Toro proved the converse: that if the estimate (1.8) holds in all bounded Lipschitz domains $V \subset \mathbb{R}^{n}$, for a given real elliptic coefficient matrix $A$, then for each such $V$ there is some $p<\infty$ such that the $L^{p}$-Dirichlet problem is well-posed in $V$. We observe that under these conditions, the BMO-Dirichlet problem is well-posed in all such domains. In the later part of [24], the authors showed that the bound (1.8) holds in two-dimensional Lipschitz domains for real $t$-independent coefficients. (In [20], the bound (1.8) was shown to hold for real $t$-independent coefficients in higher dimensions, at least in the domain above a Lipschitz graph.) Thus, Theorem 1.4 follows from [24] under the additional assumption that $A$ is real-valued. We will use these results to generalize to $A$ with small imaginary part.

In [12], Dindos, Kenig, and Pipher found another relationship between the BMO-Dirichlet problem and the $L^{p}$-Dirichlet problem for real coefficients. Specifically, they showed that if $A$ is an arbitrary coefficient matrix, elliptic and realvalued but not necessarily $t$-independent, and if $V$ is a Lipschitz domain, then the BMO-Dirichlet problem is well-posed in $V$ if and only if there is some $p$, with $1<p<\infty$, such that the $L^{p}$-Dirichlet problem is well-posed in $V$.

The outline of this paper is as follows. In Section 2 we will define our terminology and some important tools, and will review some known results. In Section 3, we will prove the existence result of Theorem 1.4; that is, we will show that for each $f \in \operatorname{BMO}(\partial V)$, there exists a function $u$ that solves the problem (1.1) and satisfies the bound (1.5). In Section 4, we will study functions $u$ that satisfy the bound (1.6); this will allow us to prove uniqueness of solutions and the converse result of Theorem 1.4, and will also allow us to prove the maximum principle (Theorem 1.7). 
The results in this paper were part of my dissertation work at the University of Chicago. I would like to thank my advisor, Carlos Kenig, for his guidance and advice. I would also like to thank Svitlana Mayboroda and the anonymous reviewer for their comments, which have greatly improved the exposition of this paper.

\section{Preliminaries}

In this section, we will begin by defining our terminology. We will then remind the reader of some known results concerning solutions to elliptic equations; we will conclude this section by discussing layer potentials, an important tool in the construction of solutions to boundary-value problems.

\subsection{Definitions}

We say that $\operatorname{div} A \nabla u=0$ in a domain $V$ in the weak sense if

$$
\int \nabla \eta \cdot A \nabla u=0 \quad \text { for all } \quad \eta \in C_{0}^{\infty}(V) .
$$

Thus, solutions $u$ to the differential equation $\operatorname{div} A \nabla u=0$ need not be smooth; they are only assumed to lie in the local Sobolev space $W_{1, l o c}^{2}(V)$ of functions with one weak derivative.

In this paper, we will work only in two-dimensional Lipschitz domains, defined as follows.

Definition 2.2. We say that the domain $\Omega \subset \mathbb{R}^{2}$ is a special Lipschitz domain if, for some Lipschitz function $\varphi$ and unit vector $\vec{e}$,

$$
\Omega=\Omega(\vec{e}, \varphi)=\left\{x \vec{e}^{\perp}+t \vec{e}: t>\varphi(x)\right\}, \quad \text { where } \quad \vec{e}^{\perp}=\left(\begin{array}{cc}
0 & 1 \\
-1 & 0
\end{array}\right) \vec{e} .
$$

We refer to $M=\left\|\varphi^{\prime}\right\|_{L^{\infty}(\mathbb{R})}$ as the Lipschitz constant of $\Omega$.

We say that the domain $V$ is a Lipschitz domain if either $V$ is a special Lipschitz domain, or if there is some positive scale $r$, some constants $M>0$ and $c_{0} \geq 1$, and some finite set $\left\{X_{j}\right\}_{j=1}^{N}$ of points with $X_{j} \in \partial V$, such that the following conditions hold. First,

$$
\partial V \subset \bigcup_{j=1}^{N} B\left(X_{j}, r_{j}\right) \text { for some } r_{j} \text { with } \frac{1}{c_{0}} r<r_{j}<c_{0} r .
$$

Second, for each $X_{j}$, there is some special Lipschitz domain $\Omega_{j}=\Omega\left(\vec{e}_{j}, \varphi_{j}\right)$ with $X_{j} \in \partial \Omega_{j}$ and with Lipschitz constant at most $M$, such that

$Z_{j} \cap \Omega_{j}=Z_{j} \cap V, \quad$ where $Z_{j}=\left\{X_{j}+x \vec{e}_{j}^{\perp}+t \vec{e}_{j}:|x|<2 r_{j},|t|<(4+4 M) r_{j}\right\}$.

If $V$ is a special Lipschitz domain let $N=c_{0}=1$; otherwise let $M, N, c_{0}$ be as above. We refer to the triple $\left(M, N, c_{0}\right)$ as the Lipschitz character of $V$.

We will let $V$ refer to an arbitrary Lipschitz domain and reserve $\Omega$ for special Lipschitz domains. If $V$ is a Lipschitz domain, we let $\sigma$ denote the surface measure on $\partial V$ and let $\nu$ denote the unit outward normal to $V$. 
We let $f$ denote the average integral $f_{E} f d \mu=\frac{1}{\mu(E)} \int_{E} f d \mu$. If $V \subset \mathbb{R}^{2}$ is a Lipschitz domain and $f$ is defined on $\partial V$, then the BMO-norm of $f$ is given by

$$
\|f\|_{\mathrm{BMO}(\partial V)}=\sup _{\Delta} \inf _{F \in \mathbb{C}}\left(f_{\Delta}|f(Y)-F|^{2} d \sigma(Y)\right)^{1 / 2},
$$

where the supremum is taken over all sets $\Delta \subseteq \partial V$ that are both bounded and connected. Observe that if $f$ is constant on each connected component of $\partial V$, then $\|f\|_{\mathrm{BMO}(\partial V)}=0$; we let $\mathrm{BMO}(\partial V)$ be the the space of all equivalence classes of functions $f$ with $\|f\|_{\mathrm{BMO}(\partial V)}<\infty$. We remind the reader that, if $\Delta \subseteq \widetilde{\Delta} \subseteq \partial V$, and if $\Delta, \widetilde{\Delta}$ are bounded connected sets, then for some absolute constant $C$,

$$
\left(f_{\widetilde{\Delta}}\left|f-f_{\Delta} f d \sigma\right|^{2} d \sigma\right)^{1 / 2} \leq\|f\|_{\operatorname{BMO}(\partial V)}(C+C \log (\sigma(\widetilde{\Delta}) / \sigma(\Delta))) .
$$

If $X \in \partial V$, we define the nontangential cone $\gamma(X)$ by

$$
\gamma(X)=\gamma_{a}(X)=\{Y \in V:|X-Y|<(1+a) \operatorname{dist}(Y, \partial V)\},
$$

where $a$ is a fixed positive number. (The exact value of $a$ is usually irrelevant provided $a>0$.) The nontangential maximal function is thus given by

$$
N u(X)=\sup _{Y \in \gamma(X)}|u(Y)|=\sup \{|u(Y)|:|X-Y|<(1+a) \operatorname{dist}(Y, \partial V)\} .
$$

By $u=f$ on $\partial V$, or $\left.u\right|_{\partial V}=f$, we mean that $f$ is the nontangential limit of $f$ on the boundary. That is, there is some $a>0$ such that, for almost every $(d \sigma)$ $X \in \partial V$, we have that

$$
\lim _{Y \rightarrow X, Y \in \gamma(X)} u(Y)=f(X) .
$$

Definition 2.8. We define a convenient set of tents $Q(X, R)$ as follows. If $X \in \partial V$, then $X \in B\left(X_{j}, r_{j}\right)$ for one of the points $X_{j}$ of Definition 2.2. Let $\vec{e}_{j}, \varphi_{j}$ be as in Definition 2.2. Then $X=x \vec{e}_{j}^{\perp}+\varphi_{j}(x) \vec{e}_{j}$ for some $x \in \mathbb{R}$. If $0<r<r_{j}$, we define

$$
Q(X, r)=\left\{y \vec{e}_{j}^{\perp}+s \vec{e}_{j}:|x-y|<r, \varphi_{j}(y)<s<\varphi_{j}(y)+(2+4 M) r .\right\}
$$

We let $\Delta(X, r)=\partial Q(X, r) \cap \partial V$.

Observe that $Q(X, r)$ is a simply connected, bounded Lipschitz domain whose Lipschitz character depends only on $M$, and that

$$
V \cap B(X, r) \subset Q(X, r) \subset V \cap B(X, C r) .
$$

Furthermore, $Q(X, r)$ is starlike with respect to the point $X+(1+2 M) r \vec{e}_{j}$.

It should be noted that $Q(X, r)$ depends on our choice of $\Omega_{j}$ and $\vec{e}_{j}$, and also that if $V$ is not a special Lipschitz domain, then $Q(X, r)$ is defined only for $r / \sigma(\partial V)$ sufficiently small. 
Finally, we will let $C$ and $\varepsilon$ denote positive constants whose value may change from line to line, but that in general depend only on the ellipticity constants $\lambda$, $\Lambda$ in formula (1.2) and on the Lipschitz character of any relevant domains $V$; any other dependency will be indicated explicitly. By $a \approx b$, we mean that for some such constant $C$, the inequalities

$$
\frac{1}{C} a \leq b \leq C a
$$

are valid.

\subsection{Bounds on solutions to elliptic equations}

In this section we discuss some well-known results concerning elliptic partial differential equations, as well as some results valid in the special case of two dimensions or of $t$-independent coefficients.

To prove Corollary 2.34 below, we will need to consider weak solutions to the inhomogeneous equation $\operatorname{div} A \nabla u=\operatorname{div} \vec{f}$, where $u \in W_{1, l o c}^{2}(V)$ is a weak solution in $V$ if

$$
\int \nabla \eta \cdot A \nabla u=\int \nabla \eta \cdot \vec{f} \text { for all } \eta \in C_{0}^{\infty}(V) .
$$

The following lemmas are thus stated in that generality. However, we will most commonly use these lemmas in the case where $\vec{f} \equiv 0$, that is, where $\operatorname{div} A \nabla u=0$.

Lemma 2.9 (The Caccioppoli inequality). Let $A$ be an elliptic matrix, and suppose that $\operatorname{div} A \nabla u=\operatorname{div} \vec{f}$ in $B(X, r)$ for some $\vec{f} \in L^{2}(B(X, r))$. Then there exists a constant $C$ depending only on the constants $\lambda$ and $\Lambda$ in formula (1.2) such that

$$
\int_{B(X, r / 2)}|\nabla u|^{2} \leq \frac{C}{r^{2}} \int_{B(X, r) \backslash B(X, r / 2)}|u|^{2}+C \int_{B(X, r)}|\vec{f}|^{2} .
$$

Remark 2.10. More generally, if $V$ is a Lipschitz domain, then for any $u$ with $\nabla u \in L^{2}(B(X, r) \cap V), \operatorname{div} A \nabla u=\operatorname{div} \vec{f}$ in $V \cap B(X, r)$ and $u=0$ on $\partial V \cap B(0, r)$, we have that

$$
\int_{B(X, r / 2) \cap V}|\nabla u|^{2} \leq \frac{C}{r^{2}} \int_{V \cap B(X, r) \backslash B(X, r / 2)}|u|^{2}+C \int_{V \cap B(X, r)}|\vec{f}|^{2}
$$

where $C$ depends only on $\lambda$ and $\Lambda$.

Lemma 2.11 (Theorem 2 in [29]). Let $A$ be elliptic, and suppose that $\operatorname{div} A \nabla u=$ $\operatorname{div} \vec{f}$ in $B(X, r)$. Then there exists a $p_{0}>2$, depending only on the constants $\lambda$ and $\Lambda$ in formula (1.2), such that if $\vec{f} \in L^{p}(B(X, r))$ for some $2<p<p_{0}$, then $\nabla u \in L_{l o c}^{p}(B(X, r))$, and

$$
\left(f_{B(X, r / 2)}|\nabla u|^{p}\right)^{1 / p} \leq C\left(f_{B(X, r)}|\nabla u|^{2}\right)^{1 / 2}+C\left(f_{B(X, r)}|\vec{f}|^{p}\right)^{1 / p} .
$$

In two dimensions, Lemmas 2.9 and 2.11 together with Morrey's inequality have an immediate corollary. 
Lemma 2.12. Let $A$ be elliptic, and suppose that $\operatorname{div} A \nabla u=\operatorname{div} \vec{f}$ in $B\left(X_{0}, r\right)$ for some $f \in L^{p}\left(B\left(X_{0}, r\right)\right), p>2$. Assume that the ambient dimension is 2 .

For some $C, \alpha>0$, depending only on $\lambda, \Lambda$ and $p$,

$$
|u(X)-u(Y)| \leq C \frac{|X-Y|^{\alpha}}{r^{\alpha}}\left\{\left(f_{B\left(X_{0}, r\right)}|u|^{2}\right)^{1 / 2}+r\left(f_{B\left(X_{0}, r\right)}|\vec{f}|^{p}\right)^{1 / p}\right\}
$$

for all $X, Y \in B\left(X_{0}, r / 2\right)$.

Furthermore,

$$
\sup _{B\left(X_{0}, r / 2\right)}|u(X)| \leq C\left(f_{B\left(X_{0}, r\right)}|u|^{2}\right)^{1 / 2}+C r\left(f_{B\left(X_{0}, r\right)}|\vec{f}|^{p}\right)^{1 / p} .
$$

Lemma 2.12 is valid in higher dimensions for real coefficients; this was first proven in [11], [32] and [31] for $A$ symmetric, and extended to nonsymmetric real equations in [30]. It is, however, not valid in higher dimensions for general complex coefficients; see [28] and [17] for specific counterexamples.

Now, suppose that $A(x, t)=A(x)$ is a $2 \times 2 t$-independent matrix. Let the matrix $B_{0}(X)$ be given by

$$
B_{0}(X)=B_{0}^{A}(X)=\left(\begin{array}{cc}
a_{11}(X) & a_{12}(X) \\
0 & 1
\end{array}\right) \text { for } A(X)=\left(\begin{array}{ll}
a_{11}(X) & a_{12}(X) \\
a_{21}(X) & a_{22}(X)
\end{array}\right)
$$

We have the following lemma from [6].

Lemma 2.16 (Lemme II.3 in [6]). If $\operatorname{div} A \nabla u=0$ in $B(X, r) \subset \mathbb{R}^{2}$, and $A(x, t)=$ $A(x)$ is t-independent, then $\operatorname{div} A \nabla u_{t}=0$ in $B(X, r)$. Furthermore, if $B_{0}$ is as in formula (2.15), then

$$
B_{0}^{A}(X) \nabla u(X)=\left(\begin{array}{l}
\tilde{u}_{t} \\
u_{t}
\end{array}\right)
$$

for some $\tilde{u}_{t}$ that satisfies $\operatorname{div} \widetilde{A} \nabla \tilde{u}_{t}=0$, for $\widetilde{A}=(1 / \operatorname{det} A) A^{T}$.

Here $A^{T}$ denotes the matrix transpose of $A$. Thus, we may apply Lemmas 2.9, 2.11 and 2.12 to the components of $B_{0}^{A} \nabla u$. In particular, by (2.14), Lemma 2.9 and the Poincaré inequality, if $\operatorname{div} A \nabla u=0$ in $B(X, r)$ then

$$
|\nabla u(X)| \leq C\left(f_{B(X, r) \backslash B(X, r / 2)}|\nabla u|^{2}\right)^{1 / 2} .
$$

\subsection{Layer potentials}

We will construct solutions to Theorem 1.4 using layer potentials, defined as follows.

If $A$ is an elliptic $2 \times 2$ matrix defined on $\mathbb{R}^{2}$, then for each $X \in \mathbb{R}^{2}$, there exists a function $\Gamma_{X}=\Gamma_{X}^{A}$ such that

$$
\int_{\mathbb{R}^{2}} A(Y) \nabla \Gamma_{X}(Y) \cdot \nabla \eta(Y) d y=-\eta(X) \text { for all } \eta \in C_{0}^{\infty}\left(\mathbb{R}^{2}\right) .
$$


This function $\Gamma_{X}$ is called the fundamental solution for $\operatorname{div} A \nabla$ with pole at $X$. It was constructed by Kenig and $\mathrm{Ni}$ in the appendix to [25] in the case of real coefficients, and by Auscher, McIntosh and Tchamitchian in Theorem 3.16 of [4] in the case of complex coefficients. (In dimensions higher than two, a construction of the fundamental solution may be found in [22].)

We will need some properties of the fundamental solution in two dimensions; see Theorem 2.6, Lemma 2.7 and Lemma 4.1 in [27] for the case where $A$ is real, and Chapter 4 of [8] for the case where $A$ is complex. If $A$ is elliptic and $t$-independent, then there is a fundamental solution $\Gamma_{X}^{A}$ that satisfies

$$
\left|\nabla \Gamma_{X}^{A}(Y)\right| \leq \frac{C}{|X-Y|}
$$

If $A$ is merely elliptic then

$$
\sup _{r>0} \int_{r \leq|X-Y| \leq 2 r}\left|\nabla \Gamma_{X}^{A}(Y)\right|^{2} d Y \leq C .
$$

If we require $\Gamma_{X}$ to satisfy the bound (2.19) or (2.20), then $\Gamma_{X}$ is unique up to an additive constant. We may choose additive constants such that, if $X, Y \in \mathbb{R}^{2}$ and $X \neq Y$, then

$$
\Gamma_{X}^{A^{T}}(Y)=\Gamma_{Y}^{A}(X)
$$

Let $V$ be a Lipschitz domain. If $f: \partial V \mapsto \mathbb{C}$ is a function, and $X \in \mathbb{R}^{2} \backslash \partial V$, the classical double layer potential $\mathcal{D} f$ is given by

$$
\mathcal{D} f(X)=\mathcal{D}_{V}^{A} f(X)=\int_{\partial V} \nu(Y) \cdot A^{T}(Y) \nabla \Gamma_{X}^{A^{T}}(Y) f(Y) d \sigma(Y) .
$$

If $A$ is discontinuous, then the conormal derivative is defined weakly, that is, by the formula

$$
\int_{\partial V} \nu(Y) \cdot A^{T}(Y) \nabla \Gamma_{X}^{A^{T}}(Y) f(Y) d \sigma(Y)=\int_{V} A^{T}(Y) \nabla \Gamma_{X}^{A^{T}}(Y) \cdot \nabla F(Y) d Y
$$

for any $F \in W_{1}^{2}(V)$ with $F=f$ on $\partial V$ and with $F \equiv 0$ near $X$. This defines $\mathcal{D} f(X)$ for all smooth, compactly supported functions $f$.

We let

$$
\mathcal{K} f(X)=\mathcal{K}_{V}^{A} f(X)=\lim _{Z \rightarrow X, Z \in \gamma(X)} \mathcal{D}_{V}^{A} f(Z)
$$

so $\mathcal{K} f=\left.\mathcal{D} f\right|_{\partial V}$ in the sense of formula (2.7). We will show (Corollary 2.34) that if $f \in \operatorname{BMO}(\partial V)$ or $f \in L^{p}(\partial V)$ for some $1<p<\infty$, then $\mathcal{K} f(X)$ exists for almost every $X \in \partial V$.

We will occasionally need a somewhat more general potential. If $\vec{f}: \partial V \mapsto \mathbb{C}^{2}$ is a vector-valued function, define

$$
\mathcal{T} \vec{f}(X)=\mathcal{T}_{V}^{A} \vec{f}(X)=\int_{\partial V} B_{0}^{A^{T}}(Y) \nabla \Gamma_{X}^{A^{T}}(Y) \cdot \vec{f}(Y) d \sigma(Y)
$$


where $B_{0}$ is as in formula (2.15). If $\left|Y-Y^{\prime}\right|<\frac{1}{2}|X-Y|$, then by Lemma 2.16 and the bounds (2.13) and (2.19),

$$
\left|B_{0}^{A^{T}}(Y) \nabla \Gamma_{X}^{A^{T}}(Y)-B_{0}^{A^{T}}\left(Y^{\prime}\right) \nabla \Gamma_{X}^{A^{T}}\left(Y^{\prime}\right)\right| \leq C \frac{\left|Y-Y^{\prime}\right|^{\alpha}}{|X-Y|^{1+\alpha}}
$$

and so the kernel $B_{0}^{A^{T}}(Y) \nabla \Gamma_{X}^{A^{T}}(Y)$ of $\mathcal{T}$ is locally Hölder continuous in $Y$. By formula (2.21) and the bound (2.13), it is locally Hölder continuous in $X$ as well. Observe that

$$
\mathcal{D} f=\mathcal{T}\left(\left(\left(B_{0}^{A^{T}}\right)^{T}\right)^{-1} A \nu f\right) .
$$

We state without proof some simple results concerning layer potentials; these results are well known in the case of harmonic functions and are straightforward to establish for the more general case. First, suppose $X \notin \partial V$ and that $1 \leq p<\infty$. Then $\mathcal{T} \vec{f}(X)$ converges for all $\vec{f} \in L^{p}(\partial V)$, and the mapping $f \mapsto \overline{\mathcal{D}} f(X)$ is well-defined and extends to a unique bounded functional on $L^{p}(\partial V)$. Also, by formula $(2.21), \operatorname{div} A \nabla(\mathcal{T} \vec{f})=0$ and $\operatorname{div} A \nabla(\mathcal{D} f)=0$ in $V$ and in $\bar{V}^{C}$.

If $V$ is bounded, then by formulas (2.18) and (2.23), D $1 \equiv 1$ in $V$ and $\mathcal{D} 1 \equiv 0$ in $\bar{V}^{C}$. More generally, if $V$ is a Lipschitz domain with compact boundary, and if $f$ is constant on each connected component of $\partial V$, then $\mathcal{D} f$ is constant in each connected component of $\mathbb{R}^{2} \backslash \partial V$. If $V=\Omega$ is a special Lipschitz domain, then $\mathcal{D} 1$ is still constant in $\Omega$ and in $\bar{\Omega}^{C}$ in the sense that, if $X, X^{\prime} \in \Omega$ or if $X, X^{\prime} \in \bar{\Omega}^{C}$, then for all $X_{0} \in \partial \Omega$,

$$
\lim _{r \rightarrow \infty} \mathcal{D} \mathbf{1}_{\partial \Omega \cap B\left(X_{0}, r\right)}(X)-\mathcal{D} \mathbf{1}_{\partial \Omega \cap B\left(X_{0}, r\right)}\left(X^{\prime}\right)=0 .
$$

Thus, by the bounds (2.19) and (2.4), if $f \in \operatorname{BMO}(\partial V)$ then $\mathcal{D} f$ is well-defined up to an additive constant.

This paper builds on the results of [8]. Specifically, we will need the following lemmas.

Lemma 2.28 (Theorem 6.1 in [8]). Let $V \subset \mathbb{R}^{2}$ be a Lipschitz domain and let $A$ be t-independent and elliptic. Suppose in addition that $A$ is smooth. Then there is some $\varepsilon_{0}>0$, depending only on the constants $\lambda, \Lambda$ in formula (1.2), such that if $\|\operatorname{Im} A\|_{L^{\infty}(\mathbb{R})}<\varepsilon_{0}$, then for any $1<p<\infty$ we have that

$$
\left\|N\left(\mathcal{T}_{V}^{A} \vec{f}\right)\right\|_{L^{p}(\partial V)} \leq C(p)\|\vec{f}\|_{L^{p}(\partial V)}
$$

where $N$ is as in formula (2.6) and where $C(p)$ depends only on $p, \lambda, \Lambda$ and the Lipschitz character of $V$.

Lemma 2.30 (Section 2.4 in [8]). Let $A$ and $V$ be as in Lemma 2.28, and suppose in addition that $\partial V$ is connected. Then there is some $\varepsilon_{1}>0$ and some $p_{0}<\infty$, depending only on $\lambda, \Lambda$ and the Lipschitz character of $V$, such that if $\|\operatorname{Im} A\|_{L^{\infty}(\mathbb{R})}<\varepsilon_{1}$, then the operator $\mathcal{K}$ is invertible on $L^{p}(\partial V)$ for any $p_{0}<p<\infty$. 
If $\mathcal{K}$ is invertible on $L^{p}(\partial V)$, then by formula (2.27) and the bound (2.29), for every $f \in L^{p}(\partial V)$ the function $u=\mathcal{D}\left(\mathcal{K}^{-1} f\right)$ exists and satisfies

$$
\left\{\begin{aligned}
\operatorname{div} A \nabla u & =0 & & \text { in } V, \\
u & =f & & \text { on } \partial V, \\
\|N u\|_{L^{p}(\partial V)} & \leq C\|f\|_{L^{p}(\partial V)} . & &
\end{aligned}\right.
$$

This is the classic method of layer potentials for constructing solutions to the Dirichlet problem. [8] used the following uniqueness result to complete the proof that the $L^{p}$-Dirichlet problem is well-posed.

Lemma 2.32 (Theorem 8.3 in [8]). Let $V \subset \mathbb{R}^{2}$ be a Lipschitz domain with connected boundary and let $A$ be $t$-independent and elliptic. Then there is some $\varepsilon_{1}>0$ and some $p_{0}<\infty$, depending only on $\lambda, \Lambda$ and the Lipschitz character of $V$, such that if $\|\operatorname{Im} A\|_{L^{\infty}(\mathbb{R})}<\varepsilon_{1}$, and if there is some $p$ with $p_{0}<p<\infty$ such that

$$
\left\{\begin{aligned}
\operatorname{div} A \nabla u & =0 & & \text { in } V, \\
u & =0 & & \text { on } \partial V, \\
N u & \in L^{p}(\partial V), & &
\end{aligned}\right.
$$

then $u \equiv 0$ in $V$.

We will also need one result concerning the behavior of $\mathcal{K}$ on $\operatorname{BMO}(\partial V)$.

Lemma 2.33 (Corollary 9.3 in [8]). Let $A$ and $V$ be as in Lemma 2.30. Then there is some $\varepsilon_{1}>0$ such that if $\|\operatorname{Im} A\|_{L^{\infty}(\mathbb{R})}<\varepsilon_{1}$, then the operator $\mathcal{K}^{*}$ is invertible on $H^{1}(\partial V)$.

Here $H^{1}(\partial V)$ denotes the Hardy space whose dual is $\mathrm{BMO}(\partial V)$, and $\mathcal{K}^{*}$ represents the operator adjoint; thus, $\mathcal{K}$ is invertible on $\operatorname{BMO}(\partial V)$ provided $\|\operatorname{Im} A\|_{L^{\infty}}$ $<\varepsilon_{1}$ and $\partial V$ is connected.

These lemmas imply that the $L^{p}$-Dirichlet problem is well-posed for smooth coefficients $A$. In [8], standard approximation techniques were used to pass to rough coefficients. In Corollary 2.34, we will use such approximation techniques to show that layer potentials are bounded, even for rough coefficients; this is somewhat more involved than the argument treating only the $L^{p}$-Dirichlet problem.

Corollary 2.34. Lemmas 2.28, 2.30 and 2.33 are valid even if $A$ is not smooth.

Furthermore, if $A$ and $V$ are as in Lemma 2.28, except that $A$ need not be smooth, then $\left.\mathcal{T} \vec{f}\right|_{\partial V}$ exists almost everywhere in $\partial V$ provided $\vec{f} \in L^{p}(\partial V)$ for some $1<p<\infty$, and $\mathcal{K} f(X)$ exists for a.e. $X \in \partial V$ provided $f \in L^{p}(\partial V)$ or $f \in \operatorname{BMO}(\partial V)$.

Proof. We wish to exploit the fact that the above lemmas are valid for smooth coefficients. Let $A_{j}(x, t)=A_{j}(x)=A * \varphi_{j}(x)$, where $\varphi_{j}(x)=j \varphi(j x)$ for some smooth, nonnegative, compactly supported function $\varphi$ with $\int_{\mathbb{R}} \varphi=1$. Observe that $A_{j}$ is smooth, $t$-independent, $\left\|\operatorname{Im} A_{j}\right\|_{L^{\infty}} \leq\|\operatorname{Im} A\|_{L^{\infty}}$, and that $A_{j}$ satisfies the ellipticity condition (1.2) with the same constants $\lambda, \Lambda$ as $A$. Furthermore, if $U$ is a bounded set and $1 \leq q<\infty$, then $A_{j} \rightarrow A$ in $L^{q}(U)$ as $j \rightarrow \infty$. 
Observe that Lemma 2.28 is valid for $A_{j}$; to show that it is valid for $A$ as well, we must control $\mathcal{T}^{A}-\mathcal{T}^{A_{j}}$. We begin by controlling the kernels of these operators. Specifically, we will begin by bounding the $L^{2}$ norm of $\nabla \Gamma_{X}^{A}-\nabla \Gamma_{X}^{A_{j}}$.

Fix some positive number $r$. Let $\vec{f}$ be a smooth vector field supported in $B(0,3 r)$. Define

$$
\begin{aligned}
u(X) & =-\int_{B(0,3 r)} \vec{f}(Y) \cdot \nabla \Gamma_{X}^{A^{T}}(Y) d Y=\int_{B(0,3 r)} \operatorname{div} \vec{f}(Y) \Gamma_{X}^{A^{T}}(Y) d Y, \\
u_{j}(X) & =-\int_{B(0,3 r)} \vec{f}(Y) \cdot \nabla \Gamma_{X}^{A_{j}^{T}}(Y) d Y .
\end{aligned}
$$

We begin with some preliminary bounds on $u$ and $\nabla u$. By the bound (2.19), we have that if $p_{1}>2$, then $|u(X)|$ is uniformly bounded and

$$
|u(X)| \leq C\left(p_{1}\right) \min \left(r^{1-2 / p_{1}}, r^{2-2 / p_{1}} \operatorname{dist}(X, \operatorname{supp} \vec{f})^{-1}\right)\|\vec{f}\|_{L^{p_{1}}(B(0, r))} .
$$

Similarly, we may bound $\nabla u$ in terms of $\operatorname{div} \vec{f}$; thus $\nabla u$ is locally in $L^{2}$ and we may apply the lemmas in Section 2.2.

By the definition (2.18) of $\Gamma_{Y}^{A}$ and by formula (2.21), we have that

$$
\begin{aligned}
\int_{\mathbb{R}^{2}} \nabla \eta(X) \cdot A(X) \nabla u(X) d X & =\int_{\mathbb{R}^{2}} \operatorname{div} \vec{f}(Y) \int_{\mathbb{R}^{2}} \nabla \eta(X) \cdot A(X) \nabla \Gamma_{Y}^{A}(X) d X d Y \\
& =-\int_{\mathbb{R}^{2}} \operatorname{div} \vec{f}(Y) \eta(Y) d Y=\int_{\mathbb{R}^{2}} \vec{f}(Y) \cdot \nabla \eta(Y) d Y
\end{aligned}
$$

and so $\operatorname{div} A \nabla u=\operatorname{div} \vec{f}$ in $\mathbb{R}^{2}$.

Thus by Lemmas 2.9 and 2.11 , we have that if $R>0$ and if $2<p_{1}<p_{0}$, where $p_{0}$ is as in Lemma 2.11, then

$$
\left(\int_{B(0, R)}|\nabla u|^{p_{1}}\right)^{1 / p_{1}} \leq \frac{C\left(p_{1}\right)}{R^{1-2 / p_{1}}}\left(f_{B(0,2 R)}|u|^{2}\right)^{1 / 2}+C\left(p_{1}\right)\left(\int_{B(0, R)}|\vec{f}|^{p_{1}}\right)^{1 / p_{1}} .
$$

We may take the limit as $R \rightarrow \infty$; this yields that

$$
\|\nabla u\|_{L^{p_{1}\left(\mathbb{R}^{2}\right)}} \leq C\|\vec{f}\|_{L^{p_{1}(B(0,3 r))}} .
$$

Now we wish to consider $u-u_{j}$. Observe that

$$
\operatorname{div} A_{j} \nabla\left(u-u_{j}\right)=\operatorname{div} A_{j} \nabla u-\operatorname{div} \vec{f}=\operatorname{div}\left(A_{j}-A\right) \nabla u .
$$

By the bound (2.14), we then have that if $|X|<R / 2$ and $2<p_{2}<p_{1}$, then

$$
\begin{aligned}
\left|u(X)-u_{j}(X)\right| \leq & C\left(f_{B(0, R)}\left|u-u_{j}\right|^{2}\right)^{1 / 2} \\
& +C R^{1-2 / p_{2}}\left(\int_{B(0, R)}\left|\left(A-A_{j}\right) \nabla u\right|^{p_{2}}\right)^{1 / p_{2}} .
\end{aligned}
$$


Fix some $\delta>0$. By our bounds on $|u|$ above, if $R$ is large enough, depending only on $r$ and $\delta$, then the first term is at most $\delta\|\vec{f}\|_{L^{p_{1}(B(0,3 r))} / 2 \text {. We may assume }}$ $R>4 r$, and so the bound

$$
\left|u(X)-u_{j}(X)\right| \leq \frac{\delta}{2}\|\vec{f}\|_{L^{p_{1}(B(0,3 r))}}+C R^{1-2 / p_{2}}\left(\int_{B(0, R)}\left|\left(A-A_{j}\right) \nabla u\right|^{p_{2}}\right)^{1 / p_{2}}
$$

is valid for all $|X|<2 r$ and all $\vec{f}$ smooth and supported in $B(0,3 r)$.

By Hölder's inequality, we have that

$$
\left(\int_{B(0, R)}\left|\left(A-A_{j}\right) \nabla u\right|^{p_{2}}\right)^{1 / p_{2}} \leq\left(\int_{B(0, R)}\left|A-A_{j}\right|^{q}\right)^{1 / q}\left(\int_{B(0, R)}|\nabla u|^{p_{1}}\right)^{1 / p_{1}}
$$

for some $q<\infty$. Recall that $A_{j} \rightarrow A$ in $L^{q}(B(0, R))$; thus, there is some $j$ depending on $R$ and $\delta$ such that

$$
\left|u(X)-u_{j}(X)\right| \leq \delta\|\vec{f}\|_{L^{p_{1}(B(0, r))}}
$$

for all $|X|<2 r$ and all $\vec{f}$ smooth and supported in $B(0,3 r)$.

But recall that

$$
u(X)-u_{j}(X)=\int_{B(0,3 r)} \vec{f} \cdot\left(\nabla \Gamma_{X}^{A_{j}^{T}}-\nabla \Gamma_{X}^{A^{T}}\right)
$$

Thus, our pointwise bound on $u-u_{j}$ implies that, if $j$ is large enough (depending only on $r$ and $\delta$ ), then

$$
\left\|\nabla \Gamma_{X}^{A}-\nabla \Gamma_{X}^{A_{j}}\right\|_{L^{p_{1}^{\prime}(B(0,3 r))}} \leq \delta
$$

for all $|X|<2 r$.

Fix some $\eta>0$. By Hölder's inequality,

$$
\begin{aligned}
\int_{B(0,3 r) \backslash B(X, \eta)}\left|\nabla \Gamma_{X}^{A^{T}}-\nabla \Gamma_{X}^{A_{j}^{T}}\right|^{2} \leq & \left(\int_{B(0,3 r) \backslash B(X, \eta)}\left|\nabla \Gamma_{X}^{A^{T}}-\nabla \Gamma_{X}^{A_{j}^{T}}\right|^{p_{1}}\right)^{1 / p_{1}} \\
& \times\left(\int_{B(0,3 r) \backslash B(X, \eta)}\left|\nabla \Gamma_{X}^{A^{T}}-\nabla \Gamma_{X}^{A_{j}^{T}}\right|^{p_{1}^{\prime}}\right)^{1 / p_{1}^{\prime}} .
\end{aligned}
$$

By the bound (2.19) the first term is at most $C(r, \eta)$, and by the above arguments, if $j$ is large enough then the second term is at most $\delta$. Thus,

$$
\left\|\nabla \Gamma_{X}^{A^{T}}-\nabla \Gamma_{X}^{A_{j}^{T}}\right\|_{L^{2}(B(0,3 r) \backslash B(X, \eta))} \leq \delta C(r, \eta) .
$$

Recall that

$$
\mathcal{T}^{A} \vec{f}(X)=\int_{\partial V} B_{0}^{A^{T}}(Y) \nabla \Gamma_{X}^{A^{T}}(Y) \cdot \vec{f}(Y) d \sigma(Y)
$$


We wish to bound the difference $B_{0}^{A^{T}} \nabla \Gamma_{X}^{A^{T}}-B_{0}^{A_{j}^{T}} \nabla \Gamma_{X}^{A_{j}^{T}}$ in the kernels of $\mathcal{T}^{A}$ and $\mathcal{T}^{A_{j}}$. Observe that

$$
\begin{aligned}
B_{0}^{A^{T}}(Y) \nabla \Gamma_{X}^{A^{T}}(Y)-B_{0}^{A_{j}^{T}}(Y) \nabla \Gamma_{X}^{A_{j}^{T}}(Y)= & \left(B_{0}^{A^{T}}(Y)-B_{0}^{A_{j}^{T}}(Y)\right) \nabla \Gamma_{X}^{A^{T}}(Y) \\
& +B_{0}^{A_{j}^{T}}(Y)\left(\nabla \Gamma_{X}^{A^{T}}(Y)-\nabla \Gamma_{X}^{A_{j}^{T}}(Y)\right)
\end{aligned}
$$

and that $B_{0}^{A_{j}^{T}} \rightarrow B_{0}^{A^{T}}$ in $L^{q}(U)$ for any $q<\infty$ and any bounded set $U$. Thus, again using Hölder's inequality and the bound (2.19) on $\left|\nabla \Gamma_{X}^{A^{T}}\right|$, we have that for any $\varepsilon>0$ and any $r>\eta>0$,

$$
\left\|B_{0}^{A^{T}} \nabla \Gamma_{X}^{A^{T}}-B_{0}^{A_{j}^{T}} \nabla \Gamma_{X}^{A_{j}^{T}}\right\|_{L^{2}(B(0,3 r) \backslash B(X, \eta))} \leq \varepsilon
$$

for all $j$ sufficiently large.

We now improve to a pointwise bound.

Choose some $r>\eta>0$ and some $X$ and $Y$ with $|X|<2 r,|Y|<2 r$ and $|X-Y|>\eta$. Define

$$
\left(\begin{array}{c}
v \\
w
\end{array}\right)=B_{0}^{A^{T}} \nabla \Gamma_{X}^{A^{T}}, \quad\left(\begin{array}{c}
v_{j} \\
w_{j}
\end{array}\right)=B_{0}^{A_{j}^{T}} \nabla \Gamma_{X}^{A_{j}^{T}}
$$

By Lemma 2.16, $\operatorname{div} \widetilde{A^{T}} \nabla v=0$ and $\operatorname{div} \widetilde{A_{j}^{T}} \nabla v_{j}=0$ away from $X$. As before,

$$
\operatorname{div} \widetilde{A_{j}^{T}} \nabla\left(v-v_{j}\right)=\operatorname{div} \widetilde{A_{j}^{T}} \nabla v=\operatorname{div}\left(\widetilde{A_{j}^{T}}-\widetilde{A^{T}}\right) \nabla v
$$

Applying the bound (2.14) to the function $v-v_{j}$, we see that if $|X-Y|>2 \eta$ and if $p_{2}>2$, then

$$
\left|v(Y)-v_{j}(Y)\right| \leq C\left(f_{B(Y, \eta / 2)}\left|v-v_{j}\right|^{2}\right)^{1 / 2}+C \eta\left(f_{B(Y, \eta)}\left|\widetilde{A^{T}}-\widetilde{A_{j}^{T}}\right|^{p_{2}}|\nabla v|^{p_{2}}\right)^{1 / p_{2}} .
$$

The first term is at most $C\left\|B_{0}^{A^{T}} \nabla \Gamma_{X}^{A^{T}}-B_{0}^{A_{j}^{T}} \nabla \Gamma_{X}^{A_{j}^{T}}\right\|_{L^{2}(B(0,3 r) \backslash B(X, \eta))}$. By the bound (2.17), Lemma 2.9 and the bound (2.19), we have that $|\nabla v| \leq C / \eta^{2}$ in $B(Y, \eta)$. Thus, since $A_{j} \rightarrow A$ in $L^{p_{2}}(B(0,3 r))$, we have that $v_{j} \rightarrow v$ uniformly in $X$ and $Y$. A similar argument is valid for $w$.

Thus, for any $\zeta>0$ and any $r>\eta>0$, we have that there is some $j$ such that

$$
\left|B_{0}^{A^{T}}(Y) \nabla \Gamma_{X}^{A^{T}}(Y)-B_{0}^{A_{j}^{T}}(Y) \nabla \Gamma_{X}^{A_{j}^{T}}(Y)\right| \leq \zeta
$$

for all $X, Y \in B(0,2 r)$ with $|X-Y|>2 \eta$.

Define

$$
N_{\eta, r} F(Z)=\sup \{|F(X)|: X \in \gamma(Z) \backslash B(Z, 2 \eta),|X|<r\} .
$$

We use the above remarks to bound $N_{\eta, r}\left(\mathcal{T}^{A}\left(\mathbf{1}_{B(0,2 r)} \vec{f}\right)\right)$. Specifically, if $j$ is large enough, then we have that

$$
\left|N_{\eta, r}\left(\mathcal{T}^{A}\left(\mathbf{1}_{B(0,2 r)} \vec{f}\right)\right)(X)\right| \leq\left|N_{\eta, r}\left(\mathcal{T}^{A_{j}}\left(\mathbf{1}_{B(0,2 r)} \vec{f}\right)\right)(X)\right|+\zeta \int_{\partial V \cap B(0,2 r)}|\vec{f}| d \sigma
$$


and so the bound

$$
\left\|N_{\eta, r}\left(\mathcal{T}^{A}\left(\mathbf{1}_{B(0,2 r)} \vec{f}\right)\right)\right\|_{L^{p}(\partial V)} \leq C(p)\|\vec{f}\|_{L^{p}(\partial V)}
$$

follows from the uniform bound on $\left\|N\left(\mathcal{T}^{A_{j}} \vec{f}\right)\right\|_{L^{p}(\partial V)}$.

Bounding $N_{\eta, r}\left(\mathcal{T}^{A}\left(\mathbf{1}_{\partial V \backslash B(0,2 r)} \vec{f}\right)\right)$ is much more straightforward. If $\partial V$ is compact then we consider only $r$ large enough that $\partial V \subset B(0,2 r)$. Otherwise, $V$ is a special Lipschitz domain. By the bound (2.19) and by the definition (2.25) of $\mathcal{T}$,

$$
\left|\mathcal{T}^{A}\left(\mathbf{1}_{\partial V \backslash B(0,2 r)} \vec{f}\right)(X)\right| \leq C r^{-1 / p}\|\vec{f}\|_{L^{p}(\partial V)} \quad \text { for all }|X|<r
$$

and so $\left\|N_{\eta, r}\left(\mathcal{T}^{A}\left(\mathbf{1}_{\partial V \backslash B(0,2 r)} \vec{f}\right)\right)\right\|_{L^{p}(\partial V)} \leq C\|\vec{f}\|_{L^{p}(\partial V)}$.

Combining these results gives the estimate

$$
\left\|N_{\eta, r}\left(\mathcal{T}^{A} \vec{f}\right)\right\|_{L^{p}(\partial V)} \leq C(p)\|\vec{f}\|_{B(0,2 r) \cap \partial V}
$$

This estimate is uniform in $\eta$ and $r$; letting $\eta \rightarrow 0$ and $r \rightarrow \infty$, we recover the same estimate on $N\left(\mathcal{T}^{A} \vec{f}\right)$.

We thus have that the bound (2.29) is valid, and so Lemma 2.28 is valid even if $A$ is not smooth.

Recall that Lemmas 2.30 and 2.33 were proven in [8] in the case where $A$ is smooth; we observe that [8] used the smoothness assumption only to establish boundedness of layer potentials, and so by the arguments therein, Lemma 2.28 implies Lemmas 2.30 and 2.33 even if $A$ is not smooth.

Finally, we establish that $\mathcal{T} \vec{f}, \mathcal{D} f$ have nontangential limits for $\vec{f} \in L^{p}(\partial V)$, $1<p<\infty$, and for $f \in \operatorname{BMO}(\partial V)$.

There is a bounded invertible matrix $B_{1}$ such that, if $\vec{g}$ is smooth and compactly supported, then $\mathcal{T}\left(B_{1} \vec{g}\right)$ has a nontangential limit at all points in $\partial V$; see Lemma 5.7 in [8]. By standard techniques, if the bound (2.29) is valid, then we may extend the condition that $\mathcal{T} \vec{f}$ has a nontangential limit at almost every point in $\partial V$ to all $\vec{f} \in L^{p}(\partial V)$, not only $\vec{f}$ of the form $\vec{f}=B_{1} \vec{g}$ for $\vec{g} \in C_{0}^{\infty}$. By formula (2.27), if $f \in L^{p}(\partial V)$ then $\mathcal{D} f$ has a nontangential limit at $X$ and so $\mathcal{K} f(X)$ exists for almost every $X \in \partial V$.

If $f \in \operatorname{BMO}(\partial V)$ and $\partial V$ is compact, then $\mathcal{K} f$ exists because $f \in L^{2}(\partial V)$. If $V=\Omega$ is a special Lipschitz domain and $f \in \operatorname{BMO}(\partial \Omega)$, then let $\Delta$ be any connected bounded subset of $\partial \Omega$. We may take $\int_{\Delta} f d \sigma=0$. Let $\widetilde{\Delta} \supsetneq \Delta$ with $\operatorname{dist}(\Delta, \partial \Omega \backslash \widetilde{\Delta})>0$. Write $f=f_{1}+f_{2}$, where $f_{1}=f$ in $\widetilde{\Delta}$ and $f_{2}=f$ in $\partial \Omega \backslash \widetilde{\Delta}$. Then $\mathcal{K} f_{1}(X)$ exists for almost every $X \in \partial \Omega$ because $f_{1} \in L^{2}(\partial \Omega)$. By the bound (2.19), formula (2.21) and the bound (2.13), if $X, Z$ are far from supp $f_{2}$ then

$$
\left|\mathcal{D} f_{2}(X)-\mathcal{D} f_{2}(Z)\right| \leq \int_{\partial \Omega} \frac{|X-Z|^{\alpha}}{|X-Y|^{1+\alpha}}\left|f_{2}(Y)\right| d \sigma(Y)
$$

and so by the bound $(2.4), \mathcal{K} f_{2}(X)$ exists for all $X \in \Delta$. This completes the proof. 


\section{Existence of solutions}

In this section, we establish the existence result in Theorem 1.4 by proving the following theorem. We will complete the proof of Theorem 1.4 in Section 4.

Theorem 3.1. Suppose that $A$ is t-independent and elliptic, and that $V \subset \mathbb{R}^{2}$ is a Lipschitz domain. Assume that the boundary layer potential $\mathcal{K}$ is bounded and invertible on $\operatorname{BMO}(\partial V)$.

Then for every $g \in \operatorname{BMO}(\partial V)$, there exists a function $u$ with $\operatorname{div} A \nabla u=0$ in $V,\left.u\right|_{\partial V}=g$, and such that

$$
\frac{1}{\sigma\left(\partial V \cap B\left(X_{0}, r\right)\right)} \int_{V \cap B\left(X_{0}, r\right)}|\nabla u(X)|^{2} \operatorname{dist}(X, \partial V) d X \leq C\|g\|_{\mathrm{BMO}}^{2},
$$

where $C$ depends only on $\lambda, \Lambda$, the Lipschitz character of $V$, and the BMO bound of $\mathcal{K}$ and $\mathcal{K}^{-1}$.

By Lemma 2.33 and Corollary 2.34, if $A$ is elliptic and $t$-independent and if $\|\operatorname{Im} A\|_{L^{\infty}}$ is small enough, and if $V$ is a Lipschitz domain with connected boundary, then the conditions of Theorem 3.1 hold.

The solution $u$ is constructed by the classic method of layer potentials, that is, by letting $u=\mathcal{D}\left(\mathcal{K}^{-1} g\right)$. If we let $f=\mathcal{K}^{-1} g$, then because $\mathcal{K}$ is invertible on $\operatorname{BMO}(\partial V)$, there is some $C$ such that $\|f\|_{\mathrm{BMO}(\partial V)} \leq C\|g\|_{\mathrm{BMO}(\partial V)}$. Thus, to prove Theorem 3.1, we need only show that the inequality

$$
\frac{1}{\sigma\left(\partial V \cap B\left(X_{0}, r\right)\right)} \int_{V \cap B\left(X_{0}, r\right)}|\nabla \mathcal{D} f(X)|^{2} \operatorname{dist}(X, \partial V) d X \leq C\|f\|_{\mathrm{BMO}(\partial V)}^{2}
$$

is true for all $f \in \operatorname{BMO}(\partial V)$. We remark that the bound (3.2) is valid without any assumptions on $\mathcal{K}$ or $\|\operatorname{Im} A\|_{L^{\infty}}$; we will prove that the bound (3.2) holds for all two-dimensional Lipschitz domains $V$ and all elliptic $t$-independent coefficient matrices $A$.

\subsection{A $L^{2}$ estimate in special Lipschitz domains}

A major step in the proof of the estimate (3.2) is the following lemma.

Lemma 3.3. Suppose that $V \subset \mathbb{R}^{2}$ is a Lipschitz domain and that $f \in L^{2}(\partial V)$. If $A$ is elliptic and t-independent, then

$$
\int_{V}|\nabla \mathcal{D} f(X)|^{2} \operatorname{dist}(X, \partial V) d X \leq C\|f\|_{L^{2}(\partial V)}^{2} .
$$

In this section, we will prove that Lemma 3.3 is valid provided $V=\Omega$ is a special Lipschitz domain; we will move to general Lipschitz domains in Section 3.2. Our proof will be by means of $T(b)$ theorems. We remark that in the case where $\Omega$ is the domain above a Lipschitz graph (that is, where $\vec{e}^{T}=(0,1)$ ), Lemma 3.3 follows from the results of Rosén in [33], and was also established by means of $T(b)$ theorems by Grau de la Herran and Hofmann in [18]. 
We will use a square-function $T(b)$ theorem of Semmes; this result is a special case of the extension (iii) on page 724 of [35].

Theorem 3.5. Suppose that $\Psi_{t}: \mathbb{R} \times \mathbb{R} \mapsto \mathbb{C}^{2 \times 2}$ is a matrix-valued function for each $t>0$, and that for some constants $\alpha>0$ and $C_{0}<\infty, \Psi_{t}$ satisfies

$$
\left|\Psi_{t}(x, y)\right| \leq C_{0} \frac{t^{\alpha}}{(t+|x-y|)^{1+\alpha}}, \quad\left|\Psi_{t}(x, y)-\Psi_{t}\left(x, y^{\prime}\right)\right| \leq C_{0} \frac{\left|y-y^{\prime}\right|^{\alpha}}{(t+|x-y|)^{1+\alpha}}
$$

whenever $\left|y-y^{\prime}\right|<|x-y| / 2$ or $\left|y-y^{\prime}\right|<t / 2$. let

If $\vec{f}: \mathbb{R} \mapsto \mathbb{C}^{2}$ is a vector-valued function, or more generally if $\vec{f}: \mathbb{R} \mapsto \mathbb{C}^{2 \times m}$,

$$
\Theta_{t} \vec{f}(x)=\int_{\mathbb{R}} \Psi_{t}(x, y) \vec{f}(y) d y
$$

Suppose that there is a constant $C_{1}<\infty$, and a matrix-valued function $b: \mathbb{R} \mapsto$ $\mathbb{C}^{2 \times 2}$, such that for all $y \in \mathbb{R}$ and all intervals $Q \subset \mathbb{R}$,

$$
|b(y)| \leq C_{1}, \quad \int_{0}^{|Q|} \int_{Q}\left|\Theta_{t} b(x)\right|^{2} \frac{d x d t}{t} \leq C_{1}|Q|, \quad\left|\left(f_{Q} b(y) d y\right)^{-1}\right| \leq C_{1} .
$$

Then there is a constant $C$ depending only on $C_{0}, C_{1}$ and $\alpha$ such that, if $\vec{f} \in$ $L^{2}\left(\mathbb{R} \mapsto \mathbb{C}^{2}\right)$, then

$$
\int_{\mathbb{R}_{+}^{2}}\left|\Theta_{t} \vec{f}(x)\right|^{2} \frac{d x d t}{t} \leq C\|\vec{f}\|_{L^{2}(\mathbb{R})}^{2}
$$

Although this theorem is sufficient for our purposes, we observe that more general results are known; see, for example, Hofmann's paper [19] for a nice survey of $T(b)$ theorems.

We reformulate this theorem in terms of special Lipschitz domains. If $\Omega=$ $\left\{x \vec{e}^{\perp}+t \vec{e}: t>\varphi(x)\right\}$, we may prove the following theorem by letting

$$
\Psi_{t}(x, y)=t \Psi\left(x \vec{e}^{\perp}+(\varphi(x)+t) \vec{e}, y \vec{e}^{\perp}+\varphi(y) \vec{e}\right)
$$

and applying Theorem 3.5.

Theorem 3.6. Let $\Omega$ be a special Lipschitz domain. Suppose that $\Psi: \Omega \times \partial \Omega \mapsto$ $\mathbb{C}^{2 \times 2}$, and that for some constants $\alpha>0$ and $C_{0}<\infty, \Psi$ satisfies

$$
\begin{gathered}
|\Psi(X, Y)| \leq C_{0} \frac{1}{|X-Y|^{2}}, \\
\left|\Psi(X, Y)-\Psi\left(X, Y^{\prime}\right)\right| \leq C_{0} \frac{\left|Y-Y^{\prime}\right|^{\alpha}}{|X-Y|^{2+\alpha}}
\end{gathered}
$$

whenever $\left|Y-Y^{\prime}\right|<\frac{1}{2}|X-Y|$.

Define $\Theta \vec{f}(X)=\int_{\partial \Omega} \Psi(X, Y) \vec{f}(Y) d \sigma(Y)$. 
Suppose that there is a constant $C_{1}<\infty$, and a matrix-valued function $b: \partial \Omega \mapsto$ $\mathbb{C}^{2 \times 2}$, such that for all $X_{0}, Y \in \partial \Omega$, all $r>0$ and all bounded connected sets $\Delta \subset \partial \Omega$

$$
\begin{aligned}
|b(Y)| & \leq C_{1}, \\
\int_{B\left(X_{0}, r\right) \cap \Omega}|\Theta b(X)|^{2} \operatorname{dist}(X, \partial \Omega) d X & \leq C_{1} r, \\
\left|\left(f_{\Delta} b(Y) d \sigma(Y)\right)^{-1}\right| & \leq C_{1} .
\end{aligned}
$$

Then there is a constant $C$, depending only on $C_{0}, C_{1}, \alpha$ and the Lipschitz constant of $\Omega$, such that if $\vec{f} \in L^{2}\left(\partial \Omega \mapsto \mathbb{C}^{2}\right)$, then

$$
\int_{\Omega}|\Theta \vec{f}(x)|^{2} \operatorname{dist}(X, \partial \Omega) d X \leq C\|\vec{f}\|_{L^{2}(\mathbb{R})} .
$$

Let $\Omega$ be a special Lipschitz domain; we now prove that Lemma 3.3 is valid with $V=\Omega$. By formula (2.27), we need only prove that

$$
\int_{\Omega}|\nabla \mathcal{T} \vec{f}(X)|^{2} \operatorname{dist}(X, \partial \Omega) d X \leq C\|\vec{f}\|_{L^{2}(\partial V)}^{2}
$$

for all $\vec{f} \in L^{2}\left(\partial \Omega \mapsto \mathbb{C}^{2}\right)$.

Recall that $\mathcal{T} \vec{f}(X)=\int_{\partial \Omega} B_{0}^{A^{T}} \nabla \Gamma_{X}^{A^{T}} \cdot \vec{f} d \sigma$. Let

$$
\Psi(X, Y)=\nabla_{X}\left(\nabla \Gamma_{X}^{A^{T}}(Y)\right)^{T} B_{0}^{A^{T}}(Y)^{T}=\nabla_{X}\left(B_{0}^{A^{T}}(Y) \nabla \Gamma_{X}^{A^{T}}(Y)\right)^{T}
$$

so that $\nabla \mathcal{T} \vec{f}(X)=\int_{\partial \Omega} \Psi(X, Y) \vec{f}(Y) d \sigma(Y)$. Defining $\Theta$ as in Theorem 3.6, we have that $\Theta \vec{f}=\nabla \mathcal{T} \vec{f}$ and so the bound (3.13) follows from the bound (3.12).

We claim that $\Psi$ satisfies the estimates (3.7) and (3.8). Let

$$
\vec{u}(X)=B_{0}^{A^{T}}(Y) \nabla \Gamma_{X}^{A^{T}}(Y) .
$$

By the bound (2.19) and formula (2.15), $|\vec{u}(X)| \leq C /|X-Y|$. By formula (2.21) and the definition (2.1) of weak solution, $\vec{u}$ satisfies $\operatorname{div} A \nabla \vec{u}=0$ away from $Y$. We use the bound (2.17) to bound $|\nabla \vec{u}(X)|$; this yields that

$$
\left|\nabla_{X}\left(B_{0}^{A^{T}}(Y) \nabla \Gamma_{X}^{A^{T}}(Y)\right)\right| \leq \frac{C}{|X-Y|^{2}}
$$

and so the estimate (3.7) holds. Similarly, the estimate (3.8) follows from the estimate (2.26).

Let

$$
b(Y)=\left(B_{0}^{A^{T}}(Y)^{T}\right)^{-1}(A(Y) \nu(Y) \quad \tau(Y))
$$

where $(A(Y) \nu(Y) \tau(Y))$ is the $2 \times 2$ matrix whose columns are $A(Y) \nu(Y)$ and $\tau(Y)$, $\nu$ is the unit outward normal vector to $\partial \Omega$, and $\tau$ is the unit tangent vector given by

$$
\nu=\left(\begin{array}{cc}
0 & 1 \\
-1 & 0
\end{array}\right) \tau
$$


We need only show that $b$ satisfies the bounds (3.9), (3.10) and (3.11) to show that the bound (3.13) is valid.

The bound (3.9) follows from the ellipticity condition (1.2) and the definition (2.15) of $B_{0}^{A^{T}}$. To establish the bound (3.10), observe that

$$
\Theta b(X)=\int_{\partial \Omega} \Psi(X, Y) b(Y) d \sigma(Y)=\nabla_{X} \int_{\partial \Omega}\left(\mathcal{D} 1(X) \quad \tau(Y) \cdot \nabla \Gamma_{X}^{A^{T}}(Y)\right) d \sigma(Y) .
$$

Recall that $\mathcal{D} 1(X)$ is constant in $\Omega$ and in $\bar{\Omega}^{C}$. Let $r$ be a large positive number. Then because $B(0, r)$ is bounded and $\Gamma_{X}$ is continuous away from $X$, we have that

$$
\int_{\partial(\Omega \cap B(0, r))} \tau(Y) \cdot \nabla \Gamma_{X}^{A^{T}}(Y) d \sigma(Y)=0 .
$$

If $X, X^{\prime} \in \Omega$, then for $r \gg|X|+\left|X^{\prime}\right|$, we have that by the bounds (2.13) and (2.19),

$$
\int_{\Omega \cap \partial B(0, r)}\left|\nabla \Gamma_{X}^{A^{T}}(Y)-\nabla \Gamma_{X^{\prime}}^{A^{T}}(Y)\right| d \sigma(Y) \leq C r \frac{\left|X-X^{\prime}\right|^{\alpha}}{r^{1+\alpha}}
$$

which approaches 0 as $r \rightarrow \infty$; thus $\int_{\partial \Omega} \tau(Y) \cdot \nabla \Gamma_{X}^{A^{T}}(Y) d \sigma(Y)$ is constant in $\Omega$. Thus, $\Theta b(X)=0$, uniformly in $\Omega$, and so the bound (3.10) holds.

3.1.1. The bound (3.11). To complete the proof of Lemma 3.3 for special Lipschitz domains, we need only show that the bound (3.11) is valid. This derivation is somewhat tedious but involves no deep theorems. Recall that the condition (3.11) states that $f_{\Delta} b(Y) d \sigma(Y)$ must be invertible for every $\Delta \subset \partial \Omega$ connected.

Choose some such $\Delta$. Let the endpoints of the segment $\Delta$ be given by $\left(x_{a}, t_{a}\right)$ and $\left(x_{b}, t_{b}\right)$; we order these two endpoints so that if $\nu$ is the unit outward normal to $\Omega$ and $\tau$ is the unit tangent vector given by formula (3.16), then $\tau$ points from $\left(x_{a}, t_{a}\right)$ to $\left(x_{b}, t_{b}\right)$. Let $\Delta_{x}=x_{b}-x_{a}, \Delta_{t}=t_{b}-t_{a}$. Thus $\sqrt{\Delta_{x}^{2}+\Delta_{t}^{2}}$ is the distance between the two endpoints. Observe that because $\Omega$ is a special Lipschitz domain, we have that $\sigma(\Delta) \approx \sqrt{\Delta_{x}^{2}+\Delta_{t}^{2}}$.

Example 3.17. As a motivating example, consider the special case where $A=I$ is the $2 \times 2$ identity matrix. Notice that $B_{0}^{I}=I$ as well, and so $b(Y)=(\nu(Y) \quad \tau(Y))$. We have that

$$
\int_{\Delta} \tau(Y) d \sigma(Y)=\left(\begin{array}{c}
\Delta_{x} \\
\Delta_{t}
\end{array}\right)
$$

a vector whose length is comparable to $\sigma(\Delta)$. Furthermore,

$$
\int_{\Delta} \nu(Y) d \sigma(Y)=\left(\begin{array}{cc}
0 & 1 \\
-1 & 0
\end{array}\right) \int_{\Delta} \tau(Y) d \sigma(Y)=\left(\begin{array}{c}
\Delta_{t} \\
-\Delta_{x}
\end{array}\right)
$$

Thus,

$$
f_{\Delta} b(Y) d \sigma(Y)=f_{\Delta}(\nu(Y) \quad \tau(Y)) d \sigma(Y)=\frac{1}{\sigma(\Delta)}\left(\begin{array}{cc}
\Delta_{t} & \Delta_{x} \\
-\Delta_{x} & \Delta_{t}
\end{array}\right) .
$$

The right-hand side is clearly invertible, and so the bound (3.11) is valid. 
We must now show that the condition (3.11) is valid even if $A$ (and thus $B_{0}^{A^{T}}$ ) is not the identity matrix. Since $b$ is bounded, it suffices to show that

$$
\left|\operatorname{det} \int_{\Delta} b(Y) d \sigma(Y)\right| \geq \sigma(\Delta)^{2} / C
$$

for some constant $C>0$. The proof will take several steps.

Our first step is to write $\int_{\Delta} b(Y) d \sigma(Y)$ in terms of the endpoints $\left(x_{a}, t_{a}\right)$ and $\left(x_{b}, t_{b}\right)$ of $\Delta$ and in terms of the matrix $A$ and its components $a_{j k}$. We will see that this integral is independent of the particular path taken from $\left(x_{a}, t_{a}\right)$ to $\left(x_{b}, t_{b}\right)$.

Lemma 3.18. We have that

$$
\int_{\Delta} b(Y) d \sigma(Y)=\left(\begin{array}{cc}
\alpha-\beta & \gamma \\
-\delta & \bar{\alpha}+\bar{\beta}
\end{array}\right)
$$

where, in the notation given above,

$$
\begin{array}{ll}
\alpha=\Delta_{t}-\int_{x_{a}}^{x_{b}}\left(\frac{a_{12}(x)}{2 a_{11}(x)}+\frac{\bar{a}_{21}(x)}{2 \bar{a}_{11}(x)}\right) d x, & \gamma=\int_{x_{a}}^{x_{b}} \frac{1}{a_{11}(x)} d x, \\
\beta=\int_{x_{a}}^{x_{b}}\left(\frac{a_{12}(x)}{2 a_{11}(x)}-\frac{\bar{a}_{21}(x)}{2 \bar{a}_{11}(x)}\right) d x, & \delta=\int_{x_{a}}^{x_{b}} \frac{\operatorname{det} A(x)}{a_{11}(x)} d x .
\end{array}
$$

and where $A(x, t)=A(x)=\left(\begin{array}{ll}a_{11}(x) & a_{12}(x) \\ a_{21}(x) & a_{22}(x)\end{array}\right)$.

Notice that if $A=I$ then $\alpha=\Delta_{t}, \gamma=\delta=\Delta_{x}$, and $\beta=0$, and so this formula is in agreement with Example 3.17.

Proof of Lemma 3.18. By assumption, $\Omega$ is a special Lipschitz domain. Recall that this means that there is some vector $\vec{e}$ and some Lipschitz function $\varphi$ such that

$$
\Omega=\left\{x \vec{e}^{\perp}+t \vec{e}: t>\varphi(x)\right\}, \quad \vec{e}^{\perp}=\left(\begin{array}{cc}
0 & 1 \\
-1 & 0
\end{array}\right) \vec{e} .
$$

Let $\vec{\psi}(x)=x \vec{e}^{\perp}+\varphi(x) \vec{e}$; then $\vec{\psi}=\left(\psi_{1}, \psi_{2}\right)$ is a parametrization of $\partial \Omega$. We have that $\left(x_{a}, t_{a}\right)=\vec{\psi}(a)$ and $\left(x_{b}, t_{b}\right)=\vec{\psi}(b)$ for some $a, b \in \mathbb{R}$.

Recall that

$$
b(Y)=\left(B_{0}^{A^{T}}(Y)^{T}\right)^{-1}(A(Y) \nu(Y) \quad \tau(Y)) .
$$

We will compute explicit formulas for $B_{0}^{A^{T}}, \nu$ and $\tau$.

First, notice that the unit tangent vector $\tau$ satisfies $\tau(\vec{\psi}(y))= \pm \vec{\psi}^{\prime}(y) /\left|\vec{\psi}^{\prime}(y)\right|$, and that the choice of signs is determined by the requirement that $\nu$ be the outward and not the inward normal vector to $\partial \Omega$. If we choose $\tau(\vec{\psi}(y))=+\vec{\psi}^{\prime}(y) /\left|\vec{\psi}^{\prime}(y)\right|$, then $\left\langle\vec{e}^{\perp}, \tau\right\rangle>0$. By formula (3.16) and the relation above between $\overrightarrow{\vec{e}}^{\perp}$ and $\vec{e}$, this implies that $\langle\vec{e}, \nu\rangle<0$, as desired. Writing explicit formulas for $\tau$ and $\nu$ in terms of the components $\psi_{1}$ and $\psi_{2}$ of $\vec{\psi}$, we see that

$$
\tau(\vec{\psi}(y))=\frac{1}{\left|\overrightarrow{\psi^{\prime}}(y)\right|}\left(\begin{array}{c}
\psi_{1}^{\prime}(y) \\
\psi_{2}^{\prime}(y)
\end{array}\right), \quad \nu(\vec{\psi}(y))=\frac{1}{\left|\overrightarrow{\psi^{\prime}}(y)\right|}\left(\begin{array}{c}
\psi_{2}^{\prime}(y) \\
-\psi_{1}^{\prime}(y)
\end{array}\right) .
$$


Next, recall from formula (2.15) that

$$
B_{0}^{A^{T}}(Y)=\left(\begin{array}{cc}
a_{11}(Y) & a_{21}(Y) \\
0 & 1
\end{array}\right) .
$$

This means that

$$
\left(B_{0}^{A^{T}}(Y)^{T}\right)^{-1}=\frac{1}{a_{11}(Y)}\left(\begin{array}{cc}
1 & 0 \\
-a_{21}(Y) & a_{11}(Y)
\end{array}\right)
$$

and

$$
\begin{aligned}
\left(B_{0}^{A^{T}}(Y)^{T}\right)^{-1} A(Y) & =\frac{1}{a_{11}(Y)}\left(\begin{array}{cc}
1 & 0 \\
-a_{21}(Y) & a_{11}(Y)
\end{array}\right)\left(\begin{array}{ll}
a_{11}(Y) & a_{12}(Y) \\
a_{21}(Y) & a_{22}(Y)
\end{array}\right) \\
& =\frac{1}{a_{11}(Y)}\left(\begin{array}{cc}
a_{11}(Y) & a_{12}(Y) \\
0 & \operatorname{det} A(Y)
\end{array}\right) .
\end{aligned}
$$

We want to evaluate $b(\vec{\psi}(y))$. We adopt the shorthand that $a_{j k}=a_{j k}(\vec{\psi}(y))$, $\operatorname{det} A=\operatorname{det} A(\vec{\psi}(y))$, and $\psi_{j}^{\prime}=\psi_{j}^{\prime}(y)$. Then

$$
\begin{aligned}
\left(B_{0}^{A^{T}}(\vec{\psi}(y))^{T}\right)^{-1} \tau(\vec{\psi}(y)) & =\frac{1}{a_{11}\left|\overrightarrow{\psi^{\prime}}(y)\right|}\left(\begin{array}{cc}
1 & 0 \\
-a_{21} & a_{11}
\end{array}\right)\left(\begin{array}{c}
\psi_{1}^{\prime} \\
\psi_{2}^{\prime}
\end{array}\right) \\
& =\frac{1}{a_{11}\left|\overrightarrow{\psi^{\prime}}(y)\right|}\left(\begin{array}{c}
\psi_{1}^{\prime} \\
-a_{21} \psi_{1}^{\prime}+a_{11} \psi_{2}^{\prime}
\end{array}\right)
\end{aligned}
$$

and

$$
\begin{aligned}
\left(B_{0}^{A^{T}}(\vec{\psi}(y))^{T}\right)^{-1} A(\vec{\psi}(y)) \nu(\vec{\psi}(y)) & =\frac{1}{a_{11}\left|\vec{\psi}^{\prime}(y)\right|}\left(\begin{array}{cc}
a_{11} & a_{12} \\
0 & \operatorname{det} A
\end{array}\right)\left(\begin{array}{c}
\psi_{2}^{\prime} \\
-\psi_{1}^{\prime}
\end{array}\right) \\
& =\frac{1}{a_{11}\left|\vec{\psi}^{\prime}(y)\right|}\left(\begin{array}{c}
a_{11} \psi_{2}^{\prime}-a_{12} \psi_{1}^{\prime} \\
-\operatorname{det} A \psi_{1}^{\prime}
\end{array}\right) .
\end{aligned}
$$

Thus,

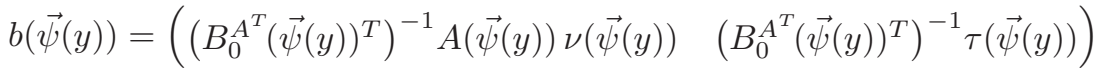

$$
\begin{aligned}
& =\frac{1}{a_{11}\left|\vec{\psi}^{\prime}(y)\right|}\left(\begin{array}{cc}
a_{11} \psi_{2}^{\prime}-a_{12} \psi_{1}^{\prime} & \psi_{1}^{\prime} \\
-\operatorname{det} A \psi_{1}^{\prime} & a_{11} \psi_{2}^{\prime}-a_{21} \psi_{1}^{\prime}
\end{array}\right) .
\end{aligned}
$$

Integrating, we see that

$$
\begin{aligned}
\int_{\Delta} b(Y) d \sigma(Y) & =\int_{a}^{b} b(\vec{\psi}(y))\left|\vec{\psi}^{\prime}(y)\right| d y \\
& =\int_{a}^{b} \frac{1}{a_{11}}\left(\begin{array}{cc}
a_{11} \psi_{2}^{\prime}-a_{12} \psi_{1}^{\prime} & \psi_{1}^{\prime} \\
-\operatorname{det} A \psi_{1}^{\prime} & a_{11} \psi_{2}^{\prime}-a_{21} \psi_{1}^{\prime}
\end{array}\right) d y \\
& =\int_{a}^{b}\left(\begin{array}{cc}
\psi_{2}^{\prime}(y) & 0 \\
0 & \psi_{2}^{\prime}(y)
\end{array}\right) d y+\int_{a}^{b} \frac{1}{a_{11}}\left(\begin{array}{cc}
-a_{12} & 1 \\
-\operatorname{det} A & -a_{21}
\end{array}\right) \psi_{1}^{\prime}(y) d y .
\end{aligned}
$$


The first integral is equal to $\Delta_{t} I$. According to our shorthand, $A$ and $a_{j k}$ are to be evaluated at $\vec{\psi}(y)$. But because $A$ is $t$-independent, $A(\vec{\psi}(y))=A\left(\psi_{1}(y)\right)$. Therefore, the second integral is equal to

$$
\int_{a}^{b} \frac{1}{a_{11}\left(\psi_{1}(y)\right)}\left(\begin{array}{cc}
-a_{12}\left(\psi_{1}(y)\right) & 1 \\
-\operatorname{det} A\left(\psi_{1}(y)\right) & -a_{21}\left(\psi_{1}(y)\right)
\end{array}\right) \psi_{1}^{\prime}(y) d y .
$$

We make the change of variables $x=\psi_{1}(y)$; thus,

$$
\int_{\Delta} b(Y) d \sigma(Y)=\left(\begin{array}{cc}
\Delta_{t} & 0 \\
0 & \Delta_{t}
\end{array}\right)+\int_{\psi_{1}(a)}^{\psi_{1}(b)} \frac{1}{a_{11}(x)}\left(\begin{array}{cc}
-a_{12}(x) & 1 \\
-\operatorname{det} A(x) & -a_{21}(x)
\end{array}\right) d x
$$

Recall that $\psi_{1}(a)=x_{a}$ and that $\psi_{1}(b)=x_{b}$. It is then elementary to compute that, if

$$
\int_{\Delta} b(Y) d \sigma(Y)=\left(\begin{array}{cc}
\alpha-\beta & \gamma \\
-\delta & \bar{\alpha}+\bar{\beta}
\end{array}\right)
$$

then

$$
\begin{array}{ll}
\alpha=\Delta_{t}-\int_{x_{a}}^{x_{b}}\left(\frac{a_{12}(x)}{2 a_{11}(x)}+\frac{\bar{a}_{21}(x)}{2 \bar{a}_{11}(x)}\right) d x, & \gamma=\int_{x_{a}}^{x_{b}} \frac{1}{a_{11}(x)} d x, \\
\beta=\int_{x_{a}}^{x_{b}}\left(\frac{a_{12}(x)}{2 a_{11}(x)}-\frac{\bar{a}_{21}(x)}{2 \bar{a}_{11}(x)}\right) d x, & \delta=\int_{x_{a}}^{x_{b}} \frac{\operatorname{det} A(x)}{a_{11}(x)} d x .
\end{array}
$$

This completes the proof.

Our goal is to control det $\int_{\Delta} b(Y) d \sigma(Y)$. We will only be able to do so in the case where $A$ is elliptic. We will need to rewrite the ellipticity condition (1.2) as a series of inequalities concerning the components $a_{j k}$ of $A$; these inequalities are the subject of the next lemma.

Lemma 3.19. Let $A=\left(\begin{array}{ll}a_{11} & a_{12} \\ a_{21} & a_{22}\end{array}\right)$. The ellipticity condition

$$
\lambda|\eta|^{2} \leq \operatorname{Re} \bar{\eta} \cdot A \eta \quad \text { for all } \eta \in \mathbb{C}^{2}
$$

is true if and only if the three inequalities

$$
\operatorname{Re} a_{11} \geq \lambda, \quad \operatorname{Re} a_{22} \geq \lambda, \quad \text { and } \quad\left|a_{12}+\bar{a}_{21}\right| \leq 2 \sqrt{\left(\operatorname{Re} a_{11}-\lambda\right)\left(\operatorname{Re} a_{22}-\lambda\right)}
$$

are valid.

Proof. We begin by making the following computation. Choose some $\eta \in \mathbb{C}^{2}$. If we let $\eta^{T}=\left(\eta_{1}, \eta_{2}\right)$, then

$$
\lambda|\eta|^{2}=\lambda\left|\eta_{1}\right|^{2}+\lambda\left|\eta_{2}\right|^{2}
$$

and

$$
\begin{aligned}
\operatorname{Re} \eta^{T} \cdot A \eta & =\operatorname{Re}\left(a_{11} \bar{\eta}_{1} \eta_{1}+a_{22} \bar{\eta}_{2} \eta_{2}+a_{12} \bar{\eta}_{1} \eta_{2}+a_{21} \bar{\eta}_{2} \eta_{1}\right) \\
& =\left|\eta_{1}\right|^{2} \operatorname{Re} a_{11}+\left|\eta_{2}\right|^{2} \operatorname{Re} a_{22}+\operatorname{Re}\left(\left(a_{12}+\bar{a}_{21}\right) \bar{\eta}_{1} \eta_{2}\right)
\end{aligned}
$$


and so

$\operatorname{Re} \eta^{T} \cdot A \eta-\lambda|\eta|^{2}=\left|\eta_{1}\right|^{2}\left(\operatorname{Re} a_{11}-\lambda\right)+\left|\eta_{2}\right|^{2}\left(\operatorname{Re} a_{22}-\lambda\right)+\operatorname{Re}\left(\left(a_{12}+\bar{a}_{21}\right) \bar{\eta}_{1} \eta_{2}\right)$.

Suppose that the three inequalities

$\operatorname{Re} a_{11} \geq \lambda, \quad \operatorname{Re} a_{22} \geq \lambda, \quad$ and $\quad\left|a_{12}+\bar{a}_{21}\right| \leq 2 \sqrt{\left(\operatorname{Re} a_{11}-\lambda\right)\left(\operatorname{Re} a_{22}-\lambda\right)}$ are valid. We then have that

$$
\begin{aligned}
\operatorname{Re} \eta^{T} \cdot A \eta-\lambda|\eta|^{2} \geq & \left|\eta_{1}\right|^{2}\left(\operatorname{Re} a_{11}-\lambda\right)+\left|\eta_{2}\right|^{2}\left(\operatorname{Re} a_{22}-\lambda\right)-\left|a_{12}+\bar{a}_{21}\right|\left|\eta_{1}\right|\left|\eta_{2}\right| \\
\geq & \left|\eta_{1}\right|^{2}\left(\operatorname{Re} a_{11}-\lambda\right)+\left|\eta_{2}\right|^{2}\left(\operatorname{Re} a_{22}-\lambda\right) \\
& -2 \sqrt{\left(\operatorname{Re} a_{11}-\lambda\right)}\left|\eta_{1}\right| \sqrt{\left(\operatorname{Re} a_{22}-\lambda\right)}\left|\eta_{2}\right| \\
= & \left(\sqrt{\left(\operatorname{Re} a_{11}-\lambda\right)}\left|\eta_{1}\right|-\sqrt{\left(\operatorname{Re} a_{22}-\lambda\right)}\left|\eta_{2}\right|\right)^{2} .
\end{aligned}
$$

The right-hand side is nonnegative for all $\eta$, and so $A$ must be elliptic.

Conversely, suppose that $A$ is elliptic, and so $\operatorname{Re} \eta^{T} \cdot A \eta-\lambda|\eta|^{2} \geq 0$ for all $\eta$. This means that

$$
\left|\eta_{1}\right|^{2}\left(\operatorname{Re} a_{11}-\lambda\right)+\left|\eta_{2}\right|^{2}\left(\operatorname{Re} a_{22}-\lambda\right)+\operatorname{Re}\left(\left(a_{12}+\bar{a}_{21}\right) \bar{\eta}_{1} \eta_{2}\right) \geq 0
$$

for any choice of $\eta_{1}$ and $\eta_{2}$.

If we choose $\eta_{1}=1$ and $\eta_{2}=0$, this implies that $\operatorname{Re} a_{11} \geq \lambda$. Similarly, if we choose $\eta_{1}=0$ and $\eta_{2}=1$ then we have that $\operatorname{Re} a_{22} \geq \lambda$.

Finally, we consider the third inequality. Choose $\left|\eta_{1}\right|^{2}=\operatorname{Re} a_{22}-\lambda+\varepsilon$ and $\left|\eta_{2}\right|^{2}=\operatorname{Re} a_{11}-\lambda+\varepsilon$ for some $\varepsilon>0$, and choose the moduli of $\eta_{1}$ and $\eta_{2}$ such that

$$
\left(a_{12}+\bar{a}_{21}\right) \bar{\eta}_{1} \eta_{2}
$$

is a negative real number. Then

$$
\begin{aligned}
0 \leq & \left|\eta_{1}\right|^{2}\left(\operatorname{Re} a_{11}-\lambda\right)+\left|\eta_{2}\right|^{2}\left(\operatorname{Re} a_{22}-\lambda\right)+\operatorname{Re}\left(\left(a_{12}+\bar{a}_{21}\right) \bar{\eta}_{1} \eta_{2}\right) \\
\leq & \left|\eta_{1}\right|^{2}\left(\operatorname{Re} a_{11}-\lambda+\varepsilon\right)+\left|\eta_{2}\right|^{2}\left(\operatorname{Re} a_{22}-\lambda+\varepsilon\right)+\operatorname{Re}\left(\left(a_{12}+\bar{a}_{21}\right) \bar{\eta}_{1} \eta_{2}\right) \\
= & 2\left(\operatorname{Re} a_{11}-\lambda+\varepsilon\right)\left(\operatorname{Re} a_{22}-\lambda+\varepsilon\right) \\
& -\left|a_{12}+\bar{a}_{21}\right| \sqrt{\left(\operatorname{Re} a_{11}-\lambda+\varepsilon\right)\left(\operatorname{Re} a_{22}-\lambda+\varepsilon\right)} .
\end{aligned}
$$

Solving, and using the fact that $\sqrt{\left(\operatorname{Re} a_{11}-\lambda+\varepsilon\right)\left(\operatorname{Re} a_{22}-\lambda+\varepsilon\right)}>0$, we see that

$$
2 \sqrt{\left(\operatorname{Re} a_{11}-\lambda+\varepsilon\right)\left(\operatorname{Re} a_{22}-\lambda+\varepsilon\right)} \geq\left|a_{12}+\bar{a}_{21}\right|
$$

for any $\varepsilon>0$; taking the limit as $\varepsilon \rightarrow 0^{+}$completes the proof.

We now use our bounds on the components $a_{j k}$ of $A$ to establish some upper and lower bounds on the quantities $\alpha, \beta, \gamma$ and $\delta$. 
Lemma 3.21. Let $\alpha, \beta, \gamma$ and $\delta$ be as in Lemma 3.18, and suppose that $A$ satisfies the ellipticity condition (1.2). We have the lower bounds

$$
\left|\Delta_{t}\right|-\left|\Delta_{x}\right| \frac{\Lambda}{\lambda} \leq|\operatorname{Re} \alpha|, \quad\left|\Delta_{x}\right| \frac{\lambda}{\Lambda^{2}} \leq|\operatorname{Re} \gamma|, \quad \text { and } \quad \lambda\left|\Delta_{x}\right| \leq|\operatorname{Re} \delta|,
$$

and the upper bounds

$$
\begin{aligned}
|\alpha| \leq\left|\Delta_{t}\right|+\left|\Delta_{x}\right| \frac{\Lambda}{\lambda}, & |\gamma| \leq \frac{1}{\lambda}\left|\Delta_{x}\right|, \\
|\beta|^{2} \leq\left(\operatorname{Re} \delta-\lambda \Delta_{x}\right)(\operatorname{Re} \gamma), & |\delta| \leq \frac{\Lambda^{2}}{\lambda}\left|\Delta_{x}\right| .
\end{aligned}
$$

Also, the real numbers $\Delta_{x}, \operatorname{Re} \gamma, \operatorname{Re} \delta$ and $\operatorname{Re} \delta-\lambda \Delta_{x}$ are either all nonpositive or all nonnegative.

Proof. By Lemma 3.19, we have that $\operatorname{Re} a_{11}>\lambda$ and $\operatorname{Re} a_{22}>\lambda$; by the ellipticity bound (1.2), we have that $\left|a_{j k}\right| \leq \Lambda$ and that $|\operatorname{det} A| \leq \Lambda^{2}$. This gives us in particular that

$$
\operatorname{Re} \frac{1}{a_{11}}=\operatorname{Re} \frac{\bar{a}_{11}}{\left|a_{11}\right|^{2}} \geq \frac{\lambda}{\Lambda^{2}}, \quad \frac{1}{\left|a_{11}\right|} \leq \frac{1}{\lambda} .
$$

Applying these inequalities gives us the two bounds on $\alpha$, the two bounds on $\gamma$ and the upper bound on $\delta$.

Notice that if $\Delta_{x}=0$, then $x_{a}=x_{b}$, and so $\beta=\gamma=\delta=0$; thus we are done. For the remainder of the proof we will assume that $\Delta_{x} \neq 0$.

The integrand in the definition of $\gamma$ is positive; therefore, $\operatorname{Re} \gamma$ and $\Delta_{x}$ are either both positive or both negative.

We are left with the lower bound on $\delta$ and the upper bound on $\beta$. Consider the integrand in the integral defining $\beta$. We compute

$$
\begin{aligned}
\left|\frac{a_{12}}{a_{11}}-\frac{\bar{a}_{21}}{\bar{a}_{11}}\right|^{2} & =\frac{a_{21} \bar{a}_{21}}{\left|a_{11}\right|^{2}}+\frac{a_{12} \bar{a}_{12}}{\left|a_{11}\right|^{2}}-\frac{a_{12} a_{21}}{\left(a_{11}\right)^{2}}-\frac{\bar{a}_{12} \bar{a}_{21}}{\left(\bar{a}_{11}\right)^{2}} \\
& =\frac{a_{21} \bar{a}_{21}+a_{12} \bar{a}_{12}}{\left|a_{11}\right|^{2}}-2 \operatorname{Re} \frac{a_{12} a_{21}}{\left(a_{11}\right)^{2}} .
\end{aligned}
$$

In order to apply Lemma 3.19, we rewrite the first fraction in terms of the quantity $\left|a_{12}+\bar{a}_{21}\right|^{2}$. Observe that

$\left|a_{12}+\bar{a}_{21}\right|^{2}=a_{12} \bar{a}_{12}+a_{21} \bar{a}_{21}+a_{12} a_{21}+\bar{a}_{12} \bar{a}_{21}=a_{12} \bar{a}_{12}+a_{21} \bar{a}_{21}+2 \operatorname{Re}\left(a_{12} a_{21}\right)$

and so

$$
\left|\frac{a_{12}}{a_{11}}-\frac{\bar{a}_{21}}{\bar{a}_{11}}\right|^{2}=\frac{\left|a_{12}+\bar{a}_{21}\right|^{2}}{\left|a_{11}\right|^{2}}-2 \operatorname{Re} \frac{a_{12} a_{21}}{\left|a_{11}\right|^{2}}-2 \operatorname{Re} \frac{a_{12} a_{21}}{\left(a_{11}\right)^{2}} .
$$

Combining the last two terms, we see that

$$
\begin{aligned}
\left|\frac{a_{12}}{a_{11}}-\frac{\bar{a}_{21}}{\bar{a}_{11}}\right|^{2} & =\frac{\left|a_{12}+\bar{a}_{21}\right|^{2}}{\left|a_{11}\right|^{2}}-2 \operatorname{Re}\left(\left(\frac{1}{\bar{a}_{11}}+\frac{1}{a_{11}}\right)\left(\frac{a_{12} a_{21}}{a_{11}}\right)\right) \\
& =\frac{\left|a_{12}+\bar{a}_{21}\right|^{2}}{\left|a_{11}\right|^{2}}-4 \operatorname{Re} \frac{1}{a_{11}} \operatorname{Re} \frac{a_{12} a_{21}}{a_{11}} .
\end{aligned}
$$


We now apply Lemma 3.19 to see that

$$
\begin{aligned}
& \left|\frac{a_{21}}{a_{11}}-\frac{\bar{a}_{12}}{\bar{a}_{11}}\right|^{2} \leq \frac{4\left(\operatorname{Re} a_{11}-\lambda\right)\left(\operatorname{Re} a_{22}-\lambda\right)}{\left|a_{11}\right|^{2}}-4 \operatorname{Re} \frac{1}{a_{11}} \operatorname{Re} \frac{a_{12} a_{21}}{a_{11}} \\
& =\frac{4 \operatorname{Re} a_{11} \operatorname{Re} a_{22}}{\left|a_{11}\right|^{2}}-\frac{4 \lambda \operatorname{Re} a_{11}}{\left|a_{11}\right|^{2}}-\frac{4 \lambda\left(\operatorname{Re} a_{22}-\lambda\right)}{\left|a_{11}\right|^{2}}-4 \operatorname{Re} \frac{1}{a_{11}} \operatorname{Re} \frac{a_{12} a_{21}}{a_{11}} .
\end{aligned}
$$

We have that

$$
\frac{\operatorname{Re} a_{11} \operatorname{Re} a_{22}}{\left|a_{11}\right|^{2}}=\frac{\operatorname{Re} a_{11}}{\left|a_{11}\right|^{2}} \operatorname{Re} \frac{a_{11} a_{22}}{a_{11}}
$$

and that

$$
\frac{\operatorname{Re} a_{11}}{\left|a_{11}\right|^{2}}=\operatorname{Re} \frac{a_{11}}{\left|a_{11}\right|^{2}}=\operatorname{Re} \frac{a_{11}}{a_{11} \bar{a}_{11}}=\operatorname{Re} \frac{1}{\bar{a}_{11}}=\operatorname{Re} \frac{1}{a_{11}} .
$$

Thus,

$$
\begin{aligned}
\left|\frac{a_{21}}{a_{11}}-\frac{\bar{a}_{12}}{\bar{a}_{11}}\right|^{2} \leq & 4 \operatorname{Re} \frac{1}{a_{11}} \operatorname{Re} \frac{a_{11} a_{22}}{a_{11}}-4 \lambda \operatorname{Re} \frac{1}{a_{11}}-\frac{4 \lambda\left(\operatorname{Re} a_{22}-\lambda\right)}{\left|a_{11}\right|^{2}} \\
& -4 \operatorname{Re} \frac{1}{a_{11}} \operatorname{Re} \frac{a_{12} a_{21}}{a_{11}} \\
= & 4 \operatorname{Re} \frac{1}{a_{11}} \operatorname{Re} \frac{\operatorname{det} A}{a_{11}}-4 \lambda \operatorname{Re} \frac{1}{a_{11}}-\frac{4 \lambda\left(\operatorname{Re} a_{22}-\lambda\right)}{\left|a_{11}\right|^{2}} .
\end{aligned}
$$

Because $\operatorname{Re} a_{22}-\lambda \geq 0$ we can disregard the final term. The left-hand side is nonnegative. Furthermore, $\operatorname{Re}\left(1 / a_{11}\right)>0$. Thus,

$$
0 \leq 4 \operatorname{Re} \frac{1}{a_{11}}\left(\operatorname{Re} \frac{\operatorname{det} A}{a_{11}}-\lambda\right) \text { and so } \operatorname{Re} \frac{\operatorname{det} A}{a_{11}} \geq \lambda .
$$

Since

$$
\delta=\int_{x_{a}}^{x_{b}} \frac{\operatorname{det} A}{a_{11}}
$$

this implies that $\Delta_{x}$ and $\operatorname{Re} \delta$ are either both positive or both negative, and moreover that $|\operatorname{Re} \delta| \geq \lambda\left|\Delta_{x}\right|$, as desired.

Finally, we have that

$$
|\beta|^{2}=\left|\int_{x_{a}}^{x_{b}} \frac{1}{2}\left(\frac{a_{21}}{a_{11}}-\frac{\bar{a}_{12}}{\bar{a}_{11}}\right)\right|^{2} .
$$

Recall that

$$
\left|\frac{a_{21}}{a_{11}}-\frac{\bar{a}_{12}}{\bar{a}_{11}}\right|^{2} \leq 4 \operatorname{Re} \frac{1}{a_{11}}\left(\operatorname{Re} \frac{\operatorname{det} A}{a_{11}}-\lambda\right)
$$

and so by Hölder's inequality,

$$
\begin{aligned}
|\beta|^{2} & \leq\left(\int_{x_{a}}^{x_{b}} \frac{1}{4}\left|\frac{a_{21}}{a_{11}}-\frac{\bar{a}_{12}}{\bar{a}_{11}}\right|^{2} \frac{1}{\operatorname{Re}\left(1 / a_{11}\right)}\right)\left(\int_{x_{a}}^{x_{b}} \operatorname{Re} \frac{1}{a_{11}}\right) \\
& \leq\left(\int_{x_{a}}^{x_{b}} \operatorname{Re} \frac{\operatorname{det} A}{a_{11}}-\lambda\right)\left(\int_{x_{a}}^{x_{b}} \operatorname{Re} \frac{1}{a_{11}}\right)=\left(\operatorname{Re} \delta-\lambda \Delta_{x}\right)(\operatorname{Re} \gamma) .
\end{aligned}
$$

This completes the proof. 
We can now establish the bound (3.11).

Lemma 3.22. The bound

$$
\operatorname{det} \int_{\Delta} b(Y) d \sigma(Y) \geq\left(\Delta_{x}^{2}+\Delta_{t}^{2}\right) / C
$$

is valid, and thus the bound (3.11) holds.

Proof. Recall from Lemma 3.18 that

$$
\int_{\Delta} b d \sigma=\left(\begin{array}{cc}
\alpha-\beta & \gamma \\
-\delta & \bar{\alpha}+\bar{\beta}
\end{array}\right)
$$

and so

$$
\operatorname{det} \int_{\Delta} b d \sigma=\alpha \bar{\alpha}-\beta \bar{\beta}+2 i \operatorname{Im}(\alpha \bar{\beta})+\gamma \delta .
$$

We use Lemma 3.21. Applying our upper bound on $\beta$, we see that

$$
\begin{aligned}
\operatorname{Redet} \int_{\Delta} b d \sigma & \geq \alpha \bar{\alpha}-\left(\operatorname{Re} \gamma \operatorname{Re} \delta-\lambda \Delta_{x} \operatorname{Re} \gamma\right)+(\operatorname{Re} \gamma \operatorname{Re} \delta-\operatorname{Im} \gamma \operatorname{Im} \delta) \\
& \geq \alpha \bar{\alpha}+\lambda \Delta_{x} \operatorname{Re} \gamma-\operatorname{Im} \gamma \operatorname{Im} \delta
\end{aligned}
$$

Applying our lower bound on $\operatorname{Re} \gamma$, we see that

$$
\operatorname{Redet} \int_{\Delta} b d \sigma \geq \alpha \bar{\alpha}+\frac{\lambda^{2}}{\Lambda^{2}} \Delta_{x}^{2}-\operatorname{Im} \gamma \operatorname{Im} \delta .
$$

We may also compute that

$$
\operatorname{Im} \operatorname{det} \int_{\Delta} b d \sigma=2 \operatorname{Im}(\alpha \bar{\beta})+\operatorname{Re} \gamma \operatorname{Im} \delta+\operatorname{Re} \delta \operatorname{Im} \gamma
$$

We wish to show that at least one of these two quantities is bounded from below. We will consider the following four cases:

- $\left|\Delta_{x}\right| \leq\left|\Delta_{t}\right|(\lambda / 4 \Lambda)$,

- $\left|\Delta_{x}\right|>\left|\Delta_{t}\right|(\lambda / 4 \Lambda),|\alpha|^{2}+\left(\lambda^{2} / 2 \Lambda^{2}\right)\left|\Delta_{x}\right|^{2} \geq \operatorname{Im} \gamma \operatorname{Im} \delta$,

- $\left|\Delta_{x}\right|>\left|\Delta_{t}\right|(\lambda / 4 \Lambda),|\alpha|^{2}+\left(\lambda^{2} / 2 \Lambda^{2}\right)\left|\Delta_{x}\right|^{2}<\operatorname{Im} \gamma \operatorname{Im} \delta$, and $|\alpha|<\left|\Delta_{x}\right| \lambda / 4 \Lambda$,

- $\left|\Delta_{x}\right|>\left|\Delta_{t}\right|(\lambda / 4 \Lambda),|\alpha|^{2}+\left(\lambda^{2} / 2 \Lambda^{2}\right)\left|\Delta_{x}\right|^{2}<\operatorname{Im} \gamma \operatorname{Im} \delta$, and $|\alpha| \geq\left|\Delta_{x}\right| \lambda / 4 \Lambda$.

Observe that these cases include all possible values of our parameters.

Suppose that $\left|\Delta_{x}\right| \leq\left|\Delta_{t}\right|(\lambda / 4 \Lambda)$. Applying our lower bound on $\alpha$ and our upper bounds on $|\gamma|$ and $|\delta|$, we see that the bound (3.23) implies that

$$
\operatorname{Redet} \int_{\Delta} b d \sigma \geq \Delta_{t}^{2}-2\left|\Delta_{t}\right|\left|\Delta_{x}\right| \frac{\Lambda}{\lambda}+\frac{\lambda^{2}}{\Lambda^{2}} \Delta_{x}^{2},
$$


and since $\left|\Delta_{x}\right| \leq\left|\Delta_{t}\right|(\lambda / 4 \Lambda)$, we have that

$$
\operatorname{Redet} \int_{\Delta} b d \sigma \geq\left|\Delta_{t}\right|^{2} / 2 \geq\left(\Delta_{x}^{2}+\Delta_{t}^{2}\right) / C
$$

as desired.

In the remaining three cases it suffices to show that $\left|\operatorname{det} \int_{\Delta} b d \sigma\right| \geq \Delta_{x}^{2} / C$. Suppose that

$$
\alpha \bar{\alpha}+\frac{\lambda^{2}}{2 \Lambda^{2}}\left|\Delta_{x}\right|^{2}-\operatorname{Im} \gamma \operatorname{Im} \delta \geq 0 .
$$

Then by formula (3.23), we have that

$$
\operatorname{Redet} \int_{\Delta} b d \sigma \geq\left|\Delta_{x}\right|^{2} \frac{\lambda^{2}}{2 \Lambda^{2}} \geq \Delta_{x}^{2} / C .
$$

Finally, suppose that $\left|\Delta_{x}\right|>\left|\Delta_{t}\right|(\lambda / 4 \Lambda)$ and $\alpha \bar{\alpha}+\left(\lambda^{2} / 2 \Lambda^{2}\right)\left|\Delta_{x}\right|^{2}<\operatorname{Im} \gamma \operatorname{Im} \delta$. In the remaining two cases we will bound $\left|\operatorname{Im} \operatorname{det} \int_{\Delta} b d \sigma\right|$ and not $\operatorname{Re} \operatorname{det} \int_{\Delta} b d \sigma$ from below. Observe that in particular, $\operatorname{Im} \delta$ and $\operatorname{Im} \gamma$ are both positive or both negative. We already know that the same is true of $\operatorname{Re} \gamma$ and $\operatorname{Re} \delta$. So by formula (3.24),

$$
\begin{aligned}
\left|\operatorname{Im} \operatorname{det} \int_{\Delta} b d \sigma\right| & \geq|\operatorname{Re} \gamma||\operatorname{Im} \delta|+|\operatorname{Re} \delta||\operatorname{Im} \gamma|-2|\alpha||\beta| \\
& \geq 2 \sqrt{\operatorname{Re} \gamma \operatorname{Re} \delta \operatorname{Im} \gamma \operatorname{Im} \delta}-2|\alpha||\beta| .
\end{aligned}
$$

Using Lemma 3.21 to bound $\beta$, and using the bound $|\alpha|^{2}+\left|\Delta_{x}\right|^{2}\left(\lambda^{2} / 2 \Lambda^{2}\right)<$ $\operatorname{Im} \gamma \operatorname{Im} \delta$, we have that

$$
\begin{aligned}
\left|\operatorname{Im} \operatorname{det} \int_{\Delta} b d \sigma\right|> & 2 \sqrt{|\alpha|^{2}+\left|\Delta_{x}^{2}\right|\left(\lambda^{2} / 2 \Lambda^{2}\right)} \sqrt{\operatorname{Re} \gamma \operatorname{Re} \delta} \\
& -2|\alpha| \sqrt{\operatorname{Re} \gamma\left(\operatorname{Re} \delta-\lambda \Delta_{x}\right)} .
\end{aligned}
$$

If $|\alpha|<\left|\Delta_{x}\right| \lambda / 4 \Lambda$, then

$$
\begin{aligned}
\left|\operatorname{Im} \operatorname{det} \int_{\Delta} b d \sigma\right| & >2 \sqrt{\left|\Delta_{x}^{2}\right|\left(\lambda^{2} / 2 \Lambda^{2}\right)} \sqrt{\operatorname{Re} \gamma \operatorname{Re} \delta}-2 \frac{\left|\Delta_{x}\right| \lambda}{4 \Lambda} \sqrt{\operatorname{Re} \gamma \operatorname{Re} \delta} \\
& \geq(\sqrt{2}-1 / 2)\left|\Delta_{x}\right| \frac{\lambda}{\Lambda} \sqrt{\operatorname{Re} \gamma \operatorname{Re} \delta}
\end{aligned}
$$

and by the lower bounds on $\operatorname{Re} \gamma$ and $\operatorname{Re} \delta$ in Lemma 3.21, the right-hand side is at least $\Delta_{x}^{2} / C$.

If $|\alpha| \geq\left|\Delta_{x}\right| \lambda / 4 \Lambda$, then

$$
\begin{aligned}
\left|\operatorname{Im} \operatorname{det} \int_{\Delta} b d \sigma\right| & >2|\alpha| \sqrt{\operatorname{Re} \gamma \operatorname{Re} \delta}-2|\alpha| \sqrt{\operatorname{Re} \gamma\left(\operatorname{Re} \delta-\lambda \Delta_{x}\right)} \\
& \geq 2|\alpha| \sqrt{\operatorname{Re} \gamma \operatorname{Re} \delta}\left(1-\sqrt{\frac{\operatorname{Re} \delta-\lambda \Delta_{x}}{\operatorname{Re} \delta}}\right) .
\end{aligned}
$$


But by Lemma 3.21,

$$
\sqrt{\frac{\operatorname{Re} \delta-\lambda \Delta_{x}}{\operatorname{Re} \delta}}=\sqrt{1-\frac{\lambda \Delta_{x}}{\operatorname{Re} \delta}} \leq \sqrt{1-\frac{\lambda^{2}}{\Lambda^{2}}}<1
$$

and so by assumption on $|\alpha|$ and, again, the lower bounds on $\operatorname{Re} \gamma$ and $\operatorname{Re} \delta$ in Lemma 3.21,

$$
\left|\operatorname{Im} \operatorname{det} \int_{\Delta} b d \sigma\right|>2|\alpha| \sqrt{\operatorname{Re} \gamma \operatorname{Re} \delta}\left(1-\sqrt{\frac{\operatorname{Re} \delta-\lambda \Delta_{x}}{\operatorname{Re} \delta}}\right) \geq \Delta_{x}^{2} / C .
$$

In all four cases, we have that $\left|\operatorname{det} \int_{\Delta} b d \sigma\right| \geq\left(\Delta_{x}^{2}+\Delta_{t}^{2}\right) / C \geq \sigma(\Delta)^{2} / C$. Thus, the bound (3.11) is valid.

This completes the proof of Lemma 3.3 in the case of special Lipschitz domains.

\subsection{A $L^{2}$ estimate in general Lipschitz domains}

We now wish to move to Lipschitz domains with compact boundary. Again by formula (2.27), to complete the proof of Lemma 3.3, we need only show that, if $V$ is a Lipschitz domain, then

$$
\int_{V}\left|\nabla \mathcal{T}_{V} \vec{f}(X)\right|^{2} \operatorname{dist}(X, \partial V) d X \leq C\|\vec{f}\|_{L^{2}(\partial V)}^{2} .
$$

Choose some Lipschitz domain $V$ with compact boundary. By Definition 2.2, there are $N$ special Lipschitz domains $\Omega_{j}$, each with Lipschitz constant at most $M$, such that

$$
\partial V \subset \bigcup_{j=1}^{N} \partial \Omega_{j} \cap B\left(X_{j}, r_{j}\right)
$$

where $r_{j}>\sigma(\partial V) / C$ and $X_{j} \in \partial V$, with $\Omega_{j} \cap B\left(X_{j}, 2 r_{j}\right)=V \cap B\left(X_{j}, 2 r_{j}\right)$.

So we may write $\vec{f}=\sum_{j=1}^{N} \vec{f}_{j}$, where $\vec{f}_{j}=0$ outside of $B\left(X_{j}, r_{j}\right)$. Pick some $j$ and note that $\mathcal{T}_{V} \vec{f}_{j} \equiv \mathcal{T}_{j} \vec{f}_{j}$, where $\mathcal{T}_{j}=\mathcal{T}_{\Omega_{j}}$. By Section 3.1,

$$
\int_{\Omega_{j}}\left|\nabla \mathcal{T}_{j} \vec{f}_{j}(X)\right|^{2} \operatorname{dist}\left(X, \partial \Omega_{j}\right) d X \leq C\left\|\vec{f}_{j}\right\|_{L^{2}\left(\partial \Omega_{j}\right)}^{2} .
$$

If $X \in B\left(X_{j}, \frac{3}{2} r_{j}\right)$, then either $\operatorname{dist}\left(X, \partial \Omega_{j}\right)=\operatorname{dist}\left(X, \partial \Omega_{j} \cap B\left(X_{j}, 2 r_{j}\right) \geq\right.$ $\operatorname{dist}(X, \partial V)$, or

$$
\operatorname{dist}\left(X, \partial \Omega_{j}\right)=\operatorname{dist}\left(X, \partial \Omega_{j} \backslash B\left(X_{j}, 2 r_{j}\right)\right)>\frac{1}{2} r_{j}>\frac{1}{3}\left|X-X_{j}\right| \geq \frac{1}{3} \operatorname{dist}(X, \partial V) .
$$

In either case $\operatorname{dist}(X, \partial V) \leq 3 \operatorname{dist}\left(X, \partial \Omega_{j}\right)$ and so

$$
\int_{V \cap B\left(X_{j}, 3 r_{j} / 2\right)}\left|\nabla \mathcal{T}_{V} \vec{f}_{j}(X)\right|^{2} \operatorname{dist}(X, \partial V) d X \leq C\left\|\vec{f}_{j}\right\|_{L^{2}\left(\partial \Omega_{j}\right)}^{2} .
$$


Conversely, suppose $X \notin B\left(X_{j}, \frac{3}{2} r_{j}\right)$. By the bound (3.14),

$$
\left|\nabla \mathcal{T}_{V} \vec{f}_{j}(X)\right| \leq \frac{C}{\left|X-X_{j}\right|^{2}} \int_{\partial V}\left|\vec{f}_{j}(Y)\right| d \sigma(Y)
$$

and because $\sigma\left(\operatorname{supp} \overrightarrow{f_{j}}\right) \leq C r_{j}$, we have that

$$
\left|\nabla \mathcal{T}_{V} \vec{f}_{j}(X)\right| \leq \frac{C}{\left|X-X_{j}\right|^{2}} \sqrt{r_{j}}\left\|\vec{f}_{j}\right\|_{L^{2}(\partial V)} .
$$

We have that $\operatorname{dist}(X, \partial V) \leq\left|X-X_{j}\right|$. Thus, we may readily compute that

$$
\begin{aligned}
\int_{V \backslash B\left(X_{j}, 3 r_{j} / 2\right)}\left|\nabla \mathcal{T}_{V} \vec{f}_{j}(X)\right|^{2} \operatorname{dist}(X, \partial V) d X \\
\leq \int_{\mathbb{R}^{2} \backslash B\left(X_{j}, 3 r_{j} / 2\right)} \frac{C}{\left|X-X_{j}\right|^{3}} r_{j}\left\|\vec{f}_{j}\right\|_{L^{2}(\partial V)}^{2} d X \leq C\left\|\vec{f}_{j}\right\|_{L^{2}(\partial V)}^{2} .
\end{aligned}
$$

Combining these estimates and summing over $j$ completes the proof of the estimate (3.25).

\subsection{BMO estimates in Lipschitz domains}

We have established that if $V$ is a Lipschitz domain and if $f \in L^{2}(\partial V)$, then

$$
\int_{V}|\nabla \mathcal{D} f(X)|^{2} \operatorname{dist}(X, \partial V) d X \leq C\|f\|_{L^{2}(\partial V)}^{2} .
$$

In this section, we show that the estimate (3.2) holds, that is, that

$$
\frac{1}{\sigma\left(\partial V \cap B\left(X_{0}, r\right)\right)} \int_{V \cap B\left(X_{0}, r\right)}|\nabla \mathcal{D} f(X)|^{2} \operatorname{dist}(X, \partial V) d X \leq C\|f\|_{\mathrm{BMO}(\partial V)}^{2}
$$

for all $f \in \operatorname{BMO}(\partial V)$, all $X_{0} \in \partial V$ and all $r>0$. This will complete the proof of Theorem 3.1. The argument comes essentially from the proof of Theorem 3 in [16], where it was applied to the Poisson extension in the upper half-space.

Choose some $r>0$ and some $X_{0} \in \partial V$. Suppose that $\partial V$ is compact and $r>\sigma(\partial V) / C$. Then $\sigma\left(\partial V \cap B\left(X_{0}, r\right)\right) \approx \sigma(\partial V)$. Recall that if $F$ is constant on each connected component of $\partial V$, then $\mathcal{D} F$ is constant in $V$; thus we may assume that $f_{\omega} f d \sigma=0$ for each connected component $\omega$ of $\partial V$. Then, by the definition (2.3) of $\operatorname{BMO}(\partial V)$,

$$
\|f\|_{L^{2}(\partial V)} \leq C \sqrt{\sigma(\partial V)}\|f\|_{\mathrm{BMO}(\partial V)}
$$

and so the bound (3.27) follows immediately from the estimate (3.26).

Otherwise, we may assume that $r$ is small enough that $Q\left(X_{0}, 2 r\right)$ exists, where the tents $Q$ are as in Definition 2.8. (If $V=\Omega$ is a special Lipschitz domain then this is true for all $r$.) Then $r \approx \sigma\left(\partial V \cap B\left(X_{0}, r\right)\right) \approx \sigma\left(\Delta\left(X_{0}, 2 r\right)\right)$. We may assume that $f_{\Delta\left(X_{0}, 2 r\right)} f d \sigma=0$. Let $\omega_{0}$ be the connected component of $\partial V$ containing $X_{0}$; we may assume that $f_{\omega} f d \sigma=0$ for any connected component $\omega$ of $\partial V$ with $\omega \neq \omega_{0}$. 
We write $f=f_{1}+f_{2}+f_{3}+f_{4}$; we define the $f_{i}$ s as follows.

If $V=\Omega$ is a special Lipschitz domain, then

$$
\begin{aligned}
& f_{1}=f \quad \text { on } \Delta\left(X_{0}, 2 r\right), \\
& f_{2}=f \quad \text { on } \partial \Omega \backslash \Delta\left(X_{0}, 2 r\right), \\
& f_{3}=f_{4}=0 .
\end{aligned}
$$

Otherwise, $\partial V$ is compact. Let $k$ be the largest integer such that $Q\left(X_{0}, 2^{k} r\right)$ exists; observe that $\sigma(\partial V) / C \leq 2^{k} r \leq \sigma(\partial V)$. Then let

$$
\begin{array}{ll}
f_{1}=f & \text { on } \Delta\left(X_{0}, 2 r\right), \\
f_{2}=f & \text { on } \Delta\left(X_{0}, 2^{k} r\right) \backslash \Delta\left(X_{0}, 2 r\right), \\
f_{3}=f & \text { on } \omega_{0} \backslash \Delta\left(X_{0}, 2^{k} r\right), \\
f_{4}=f & \text { on } \partial V \backslash \omega_{0} .
\end{array}
$$

By our definition (2.3) of BMO, we have that both $f_{1}$ and $f_{4}$ are in $L^{2}(\partial V)$, with $\left\|f_{1}\right\|_{L^{2}(\partial V)} \leq C r^{1 / 2}\|f\|_{\mathrm{BMO}(\partial V)}$ and $\left\|f_{4}\right\|_{L^{2}(\partial V)} \leq C \sigma(\partial V)^{1 / 2}\|f\|_{\mathrm{BMO}(\partial V)}$.

The bound (3.26) immediately yields that

$$
\frac{1}{r} \int_{V \cap B\left(X_{0}, r\right)}\left|\nabla \mathcal{D} f_{1}(X)\right|^{2} \operatorname{dist}(X, \partial V) d X \leq \frac{C}{r}\left\|f_{1}\right\|_{L^{2}(\partial V)}^{2} \leq C\|f\|_{\mathrm{BMO}(\partial V)}^{2} .
$$

Recall that

$$
\nabla \mathcal{D} f(X)=\int_{\partial V} \nu(Y) \cdot A^{T}(Y) \nabla\left(\nabla_{X} \Gamma_{X}^{A^{T}}(Y)\right) f(Y) d \sigma(Y)
$$

We will in fact be able to bound $\nabla \mathcal{D}\left(f-f_{1}\right)(X)$ pointwise. By the bound $(3.14)$ on $\nabla\left(\nabla_{X} \Gamma_{X}^{A^{T}}(Y)\right)$, if $X \in B\left(X_{0}, r\right)$, then

$$
\left|\nabla \mathcal{D} f_{4}(X)\right| \leq \frac{C}{\sigma(\partial V)^{2}} \int_{\partial V}\left|f_{4}\right| d \sigma \leq \frac{C}{\sigma(\partial V)^{3 / 2}}\left\|f_{4}\right\|_{L^{2}(\partial V)} \leq \frac{C}{\sigma(\partial V)}\|f\|_{\mathrm{BMO}(\partial V)} .
$$

By the bound (2.4), we have that

$$
\left\|f_{3}\right\|_{L^{2}(\partial V)} \leq \sqrt{\sigma(\partial V)}\left(C+C \log \frac{\sigma\left(\omega_{0}\right)}{\sigma(\Delta)}\right)\|f\|_{\mathrm{BMO}(\partial V)} \leq C k \sqrt{\sigma(\partial V)}\|f\|_{\mathrm{BMO}(\partial V)} .
$$

Thus, recalling that $2^{k} r \approx \sigma(\partial V)$,

$$
\left|\nabla \mathcal{D} f_{3}(X)\right| \leq \frac{C}{\left(2^{k} r\right)^{3 / 2}}\left\|f_{3}\right\|_{L^{2}(\partial V)} \leq \frac{C k}{\left(2^{k} r\right)}\|f\|_{\mathrm{BMO}(\partial V)} \leq \frac{C}{r}\|f\|_{\mathrm{BMO}(\partial V)} .
$$

Finally,

$$
\begin{aligned}
\left|\nabla \mathcal{D} f_{2}(X)\right| & \leq \sum_{j=2}^{k} \int_{\Delta\left(X_{0}, 2^{j} r\right) \backslash \Delta\left(X_{0}, 2^{j-1} r\right)}\left|\nu \cdot A^{T} \nabla\left(\nabla_{X} \Gamma_{X}^{A^{T}}\right)\right||f| d \sigma \\
& \leq \sum_{j=2}^{k} \frac{C}{\left(2^{j} r\right)^{3 / 2}}\|f\|_{L^{2}\left(\Delta\left(X_{0}, 2^{j} r\right)\right)}
\end{aligned}
$$


and again using the bound (2.4), we have that

$$
\left|\nabla \mathcal{D} f_{2}(X)\right| \leq \sum_{j=2}^{k} \frac{C j}{\left(2^{j} r\right)}\|f\|_{\mathrm{BMO}(\partial V)} \leq \frac{C}{r}\|f\|_{\mathrm{BMO}(\partial V)} .
$$

Thus, $\left|\nabla \mathcal{D}\left(f_{1}+f_{2}+f_{3}\right)(X)\right| \leq(C / r)\|f\|_{\mathrm{BMO}(\partial V)}$ for all $X \in B\left(X_{0}, r\right)$, and so

$$
\frac{1}{r} \int_{V \cap B\left(X_{0}, r\right)}\left|\nabla \mathcal{D}\left(f_{1}+f_{2}+f_{3}\right)(X)\right|^{2} \operatorname{dist}(X, \partial V) d X \leq C\|f\|_{\mathrm{BMO}(\partial V)}^{2}
$$

as desired.

Thus the bound (3.27) is valid and Theorem 3.1 is proven.

\section{Converses and uniqueness}

In this section, we will complete the proof of Theorem 1.4 by proving the following two theorems.

Theorem 4.1. Suppose that $A$ is t-independent and elliptic, and that $V \subset \mathbb{R}^{2}$ is a Lipschitz domain.

Then there is some $\varepsilon>0$ and some $C>0$, depending only on the Lipschitz character of $V$ and the constants $\lambda, \Lambda$ in the ellipticity condition (1.2), such that if $\|\operatorname{Im} A\|_{L^{\infty}}<\varepsilon$, then the following holds.

Suppose that $u$ satisfies $\operatorname{div} A \nabla u=0$ in $V$ and

$$
\sup _{X_{0} \in \partial V} \sup _{r>0} \frac{1}{r} \int_{V \cap B\left(X_{0}, r\right)}|\nabla u(X)|^{2} \operatorname{dist}(X, \partial V) d X \leq \widetilde{C}^{2} .
$$

Then $f=\left.u\right|_{\partial V}$ exists and lies in $\operatorname{BMO}(\partial V)$, with $\|f\|_{\mathrm{BMO}} \leq C \widetilde{C}$.

Theorem 4.3. Suppose $u, A$, and $V$ are as in Theorem 4.1. There is some $\varepsilon>0$ such that, if $\|\operatorname{Im} A\|_{L^{\infty}}<\varepsilon$, if $u$ satisfies

$$
\sup _{X_{0} \in \partial V} \sup _{r>0} \frac{1}{\sigma\left(\partial V \cap B\left(X_{0}, r\right)\right)} \int_{V \cap B\left(X_{0}, r\right)}|\nabla u(X)|^{2} \operatorname{dist}(X, \partial V) d X \leq \widetilde{C}^{2},
$$

and if $\left.u\right|_{\partial V}$ is a constant, then $u$ is constant.

We will also prove the maximum principle (Theorem 1.7).

Remark 4.5. We comment on the two conditions (4.2) and (4.4). First, observe that if $V$ is a Lipschitz domain then $\sigma\left(\partial V \cap B\left(X_{0}, r\right)\right) \leq C r$, and so the condition (4.4) implies that the bound (4.2) is valid at a cost of increasing $\widetilde{C}$. Conversely, if $V$ is a bounded or special Lipschitz domain, then the condition (4.2) implies that the bound (4.4) is valid at the same cost. We thus only need the more complicated formulation (4.4) if $V^{C}$ is bounded. By considering the function $u(X)=\log |X|$, which is harmonic in $V=\mathbb{R}^{2} \backslash B(0,1)$ and satisfies the bound (4.2) but not (4.4), we see that for such domains the condition (4.4) is necessary to ensure uniqueness. 
We begin by showing that the bound (4.2) is valid in all subdomains of $V$.

Lemma 4.6. Let $u$ be defined in some domain $V$. If

$$
\sup _{X_{0} \in \partial V} \sup _{r>0} \frac{1}{r} \int_{V \cap B\left(X_{0}, r\right)}|\nabla u(X)|^{2} \operatorname{dist}(X, \partial V) d X \leq \widetilde{C}^{2},
$$

and if $U \subset V$, then

$$
\sup _{X_{0} \in \partial U} \sup _{r>0} \frac{1}{r} \int_{U \cap B\left(X_{0}, r\right)}|\nabla u(X)|^{2} \operatorname{dist}(X, \partial U) d X \leq 3 \widetilde{C}^{2} .
$$

Proof. Notice that if $X_{0} \in \partial V \cap \partial U$, then the lemma follows immediately from the fact that $U \subset V$ and that $\operatorname{dist}(X, \partial U) \leq \operatorname{dist}(X, \partial V)$. Thus the only complication is the case where $X_{0} \in \partial U \backslash \partial V$.

Choose some $X_{0} \in \partial U$, and let $r>0$. Let $X_{0}^{*} \in \partial V$ satisfy $\left|X_{0}-X_{0}^{*}\right|=$ $\operatorname{dist}\left(X_{0}, \partial V\right)$. Let $R=\left|X_{0}-X_{0}^{*}\right|+r$, so $B\left(X_{0}, r\right) \subseteq B\left(X_{0}^{*}, R\right)$.

If $R \leq 3 r$, then because $\operatorname{dist}(X, \partial U) \leq \operatorname{dist}(X, \partial V)$ for all $X \in U$, we have that

$$
\frac{1}{r} \operatorname{dist}(X, \partial U) \leq \frac{3}{R} \operatorname{dist}(X, \partial V) \text {. }
$$

Conversely, if $R>3 r$ then $\left|X_{0}-X_{0}^{*}\right|>2 r$, and so for every $X \in B\left(X_{0}, r\right)$ we have that

$$
\frac{1}{r} \operatorname{dist}(X, \partial U) \leq \frac{\left|X-X_{0}\right|}{r}<1
$$

But by the triangle inequality, $\operatorname{dist}\left(X_{0}, \partial V\right) \leq\left|X-X_{0}\right|+\operatorname{dist}(X, \partial V)$, and so

$$
\frac{1}{r} \operatorname{dist}(X, \partial U)<1 \leq \frac{\operatorname{dist}(X, \partial V)}{\operatorname{dist}\left(X_{0}, \partial V\right)-\left|X-X_{0}\right|} \leq \frac{\operatorname{dist}(X, \partial V)}{R-2 r} \leq \frac{3}{R} \operatorname{dist}(X, \partial V) .
$$

In either case,

$$
\frac{1}{r} \int_{U \cap B\left(X_{0}, r\right)}|\nabla u(X)|^{2} \operatorname{dist}(X, \partial U) d X \leq \frac{3}{R} \int_{V \cap B\left(X_{0}^{*}, R\right)}|\nabla u(X)|^{2} \operatorname{dist}(X, \partial V) d X .
$$

This completes the proof.

In order to prove Theorem 4.1, we will need to show, first, that $f=\left.u\right|_{\partial V}$ exists in the sense of nontangential limits, and, second, that $f \in \operatorname{BMO}(\partial V)$. We begin with the following Fatou-type theorem.

Lemma 4.7. Let $V \subset \mathbb{R}^{2}$ be a bounded, starlike Lipschitz domain. Suppose that $A$ is $t$-independent and elliptic. There is some $\varepsilon>0$ and some $p$ with $1<p<\infty$, depending only on the constants $\lambda, \Lambda$ in formula (1.2) and the Lipschitz character of $V$, such that the following holds. If $\|\operatorname{Im} A\|_{L^{\infty}}<\varepsilon$, if $\operatorname{div} A \nabla u=0$ in $V$, and if $N u \in L^{p}(\partial V)$, then $u$ has a nontangential limit almost everywhere in $\partial V$, and $f=\left.u\right|_{\partial V}$ lies in $L^{p}(\partial V)$. 
Here the Lipschitz domain $V$ is starlike with respect to the point $Z$ if we may write $V=\{Z+r \theta:|\theta|=1,0 \leq r<\varphi(\theta)\}$ for some function $\varphi$ that is Lipschitz on the unit circle. We work in starlike domains for notational convenience and because we will apply these results only in such domains; it is possible to show that both Lemma 4.7 and Lemma 4.8 below are valid in more general domains.

Proof of Lemma 4.7. We will construct a function $\vec{h}: \partial V \mapsto \mathbb{C}^{2}$ such that $u=\mathcal{T}_{V} \vec{h}$. By Corollary 2.34, $\mathcal{T}_{V} \vec{h}$ has a nontangential limit almost everywhere in $\partial V$, so the same must be true of $u$. This is analogous to the proof of Fatou's theorem in the unit disk, with the layer potential $\mathcal{T}_{V}$ replacing the Poisson kernel.

We construct $\vec{h}$ as follows. For notational convenience, suppose that $V$ is starlike with respect to the origin. Let $V_{\delta}=(1-\delta) V$. Then $V_{\delta}$ is also a bounded Lipschitz domain whose Lipschitz character is equal to that of $V$.

Let $\mathcal{K}_{\delta}=\mathcal{K}_{V_{\delta}}, \mathcal{D}_{\delta}=\mathcal{D}_{V_{\delta}}, \mathcal{T}_{\delta}=\mathcal{T}_{V_{\delta}}$, and let $N_{\delta}$ be as in formula (2.6) with $V$ replaced by $V_{\delta}$. By Lemmas 2.30 and 2.32 and by Corollary 2.34, there is some $\varepsilon>0$ and some $p_{0}<\infty$ such that if $\|\operatorname{Im} A\|_{L^{\infty}}<\varepsilon$ and if $p_{0}<p<\infty$, then $\mathcal{K}_{\delta}$ is invertible on $L^{p}\left(\partial V_{\delta}\right)$, and such that solutions $u$ to the Dirichlet problem (1.1) that satisfy $N_{\delta} u \in L^{p}\left(\partial V_{\delta}\right)$ are unique.

For each $Y \in \bar{V}$ define $Y_{\delta}=(1-\delta) Y$. Then $\left|Y-Y_{\delta}\right| \leq \delta \operatorname{diam} V$. Because $V$ is starlike, we have that $Y_{\delta} \in V$ for any $\delta>0$ and any $Y \in \bar{V}$. If the aperture $a$ in the definition (2.5) of nontangential cone is large enough, then $Y_{\delta} \in \gamma(Y)$ for all $Y \in \partial V$ and all $\delta>0$ small enough. It is well known that we may assume the aperture to be as large as we like: if

$$
N_{a} u(X)=\sup \{|u(Y)|:|X-Y|<(1+a) \operatorname{dist}(Y, \partial V)\}
$$

and if $0<a<b$ and $0<p \leq \infty$, then

$$
\left\|N_{b} u\right\|_{L^{p}(\partial V)} \leq C(a, b)\left\|N_{a} u\right\|_{L^{p}(\partial V)} .
$$

This was proven by Fefferman and Stein for the case $V=\mathbb{R}_{+}^{n}$ (see Lemma 1 in Section 7 of [16]), and generalizes easily to arbitrary Lipschitz domains (see, for example, Lemma 3.2 in [8]).

Let $f_{\delta}\left(Y_{\delta}\right)=u\left(Y_{\delta}\right)$, so $f_{\delta}$ is defined on $\partial V_{\delta}$. Then $\left|f_{\delta}\left(Y_{\delta}\right)\right| \leq N u(Y)$, so $f_{\delta} \in L^{p}\left(\partial V_{\delta}\right)$. If $\|\operatorname{Im} A\|_{L^{\infty}}<\varepsilon$ and if $p_{0}<p<\infty$, then there is some $g_{\delta} \in L^{p}\left(\partial V_{\delta}\right)$ such that $\mathcal{K}_{\delta} g_{\delta}=f_{\delta}$. By Lemma $2.32, u=\mathcal{D}_{\delta} g_{\delta}$ in $V_{\delta}$.

Recall that by formula $(2.27), \mathcal{D} g=\mathcal{T}\left(\left(\left(B_{0}^{A^{T}}\right)^{T}\right)^{-1} A \nu g\right)$. Define

$$
\vec{g}_{\delta}\left(Y_{\delta}\right)=\left(\left(B_{0}^{A^{T}}\left(Y_{\delta}\right)\right)^{T}\right)^{-1} A\left(Y_{\delta}\right) \nu(Y) g_{\delta}\left(Y_{\delta}\right)
$$

so that $u=\mathcal{T}_{\delta} \vec{g}_{\delta}$ in $V_{\delta}$.

Define $\vec{h}_{\delta}(Y)=(1-\delta) \vec{g}_{\delta}\left(Y_{\delta}\right)$. Observe that $\left\|\vec{h}_{\delta}\right\|_{L^{p}(\partial V)} \leq C\|N u\|_{L^{p}(\partial V)}$. Choose some $X \in V$. If $\delta$ is small enough, then

$$
\begin{aligned}
\mathcal{T}_{V} \vec{h}_{\delta}(X)-u(X) & =\mathcal{T}_{V} \vec{h}_{\delta}(X)-\mathcal{T}_{\delta} \vec{g}_{\delta}(X) \\
& =\int_{\partial V}\left(B_{0}^{A^{T}}(Y) \nabla \Gamma_{X}^{A^{T}}(Y)-B_{0}^{A^{T}}\left(Y_{\delta}\right) \nabla \Gamma_{X}^{A^{T}}\left(Y_{\delta}\right)\right) \cdot \vec{h}_{\delta}(Y) d \sigma(Y) .
\end{aligned}
$$


By the bound (2.26), if $\delta \operatorname{diam} V<\frac{1}{2} \operatorname{dist}(X, \partial V)$, then the right-hand side has norm at most

$$
\delta^{\alpha} \frac{C \operatorname{diam}(V)^{\alpha}}{\operatorname{dist}(X, \partial V)^{1+\alpha}} \int_{\partial V}\left|\vec{h}_{\delta}(Y)\right| d \sigma(Y),
$$

and so $u(X)=\lim _{\delta \rightarrow 0} \mathcal{T}_{V} \vec{h}_{\delta}(X)$. The sequence $\left\{\vec{h}_{1 / n}\right\}_{n=1}^{\infty}$ is bounded in $L^{p}(\partial V)$, and so there is some subsequence that converges weakly to some function $\vec{h} \in$ $L^{p}(\partial V)$. But then

$$
\begin{aligned}
u(X) & =\lim _{k \rightarrow \infty} \mathcal{T}_{V} \vec{h}_{1 / n_{k}}(X)=\lim _{k \rightarrow \infty} \int_{\partial V} B_{0}^{A^{T}}(Y) \nabla \Gamma_{X}^{A^{T}}(Y) \cdot \vec{h}_{1 / n_{k}}(Y) d \sigma(Y) \\
& =\int_{\partial V} B_{0}^{A^{T}}(Y) \nabla \Gamma_{X}^{A^{T}}(Y) \cdot \vec{h}(Y) d \sigma(Y)=\mathcal{T}_{V} \vec{h}(X)
\end{aligned}
$$

and so $u=\mathcal{T}_{V} \vec{h}$ for some $\vec{h} \in L^{p}(\partial V)$. This completes the proof.

We now prove a lemma that relates the Carleson estimate (4.2) to nontangential maximal estimates; compare to the bound (1.8) for real coefficients.

Lemma 4.8. Suppose that $V$ is a bounded, starlike Lipschitz domain, that $A$ is $t$-independent and elliptic, that $\operatorname{div} A \nabla u=0$ in $V$ and that $u$ satisfies the condition (4.2).

There is some $\varepsilon>0$ such that, if $\|\operatorname{Im} A\|_{L^{\infty}}<\varepsilon$, then for every $X_{0} \in V$ and every $1 \leq p<\infty$, we have that

$$
\left(f_{\partial V} N\left(u-u\left(X_{0}\right)\right)^{p} d \sigma\right)^{1 / p} \leq C(p) \widetilde{C} \frac{\sigma(\partial V)}{\operatorname{dist}\left(X_{0}, \partial V\right)} .
$$

Proof. By Hölder's inequality we need only prove the estimate (4.9) for $p$ large. We begin by establishing the bound (4.9) in the case that $u=\mathcal{D} f$ for some $f \in$ $\operatorname{BMO}(\partial V)$. Once we have done this, we will work as in the proof of Lemma 4.7 to generalize to arbitrary $u$ that satisfy the bound (4.2).

Since $\partial V$ is compact, by the John-Nirenberg inequality (see, for example, page 144 of [36]), if $1 \leq p<\infty$, then

$$
\left\|f-f_{\partial V} f\right\|_{L^{p}(\partial V)} \leq C(p)\|f\|_{\mathrm{BMO}(\partial V)} \sigma(\partial V)^{1 / p} .
$$

By formula (2.27), Lemma 2.28 and Corollary 2.34, if $\|\operatorname{Im} A\|_{L^{\infty}}$ is small enough then $\|N(\mathcal{D} f)\|_{L^{p}(\partial V)} \leq C(p)\|f\|_{L^{p}(\partial V)}$ for all $1<p<\infty$. Furthermore, since $V$ is bounded, $\mathcal{D} 1 \equiv 1$ in $V$. Therefore,

$$
\begin{aligned}
\left\|N\left(\mathcal{D} f-f_{\partial V} f\right)\right\|_{L^{p}(\partial V)} & =\left\|N\left(\mathcal{D}\left(f-f_{\partial V} f\right)\right)\right\|_{L^{p}(\partial V)} \leq C(p)\left\|f-f_{\partial V} f\right\|_{L^{p}(\partial V)} \\
& \leq C(p)\|f\|_{\operatorname{BMO}(\partial V)} \sigma(\partial V)^{1 / p} .
\end{aligned}
$$

We can bound $\mathcal{D} f-f_{\partial V} f$ another way: if $X_{0} \in V$, then by the bound (2.19),

$$
\begin{aligned}
\left|\mathcal{D} f\left(X_{0}\right)-f_{\partial V} f\right| & =\left|\mathcal{D}\left(f-f_{\partial V} f\right)\left(X_{0}\right)\right|=\left|\int_{\partial V} \nu \cdot A^{T} \nabla \Gamma_{X_{0}}^{A^{T}}\left(f-f_{\partial V} f\right) d \sigma\right| \\
& \leq \frac{C \sigma(\partial V)}{\operatorname{dist}\left(X_{0}, \partial V\right)} f_{\partial V}\left|f-f_{\partial V} f\right| d \sigma \leq \frac{C \sigma(\partial V)}{\operatorname{dist}\left(X_{0}, \partial V\right)}\|f\|_{\mathrm{BMO}(\partial V)} .
\end{aligned}
$$


Thus,

$$
\left\|N\left(\mathcal{D} f-\mathcal{D} f\left(X_{0}\right)\right)\right\|_{L^{p}(\partial V)} \leq C \sigma(\partial V)^{1 / p} \frac{\sigma(\partial V)}{\operatorname{dist}\left(X_{0}, \partial V\right)}\|f\|_{\operatorname{BMO}(\partial V)} .
$$

To complete the proof of the bound (4.9) in the special case where $u=\mathcal{D} f$, we need only show that $\|f\|_{\mathrm{BMO}(\partial V)} \leq C \widetilde{C}$. (We remark that the reverse inequality is valid and is simply the bound (3.2) of Section 3.)

As shown in Section 3 of [24], if $A=A_{0}$ for some real matrix $A_{0}$, then Lemma 4.8 is valid. We wish to exploit this fact.

Let $A_{0}=\operatorname{Re} A$, and let $\mathcal{D}_{0}=\mathcal{D}_{V}^{A_{0}}, \mathcal{K}_{0}=\mathcal{K}_{V}^{A_{0}}$ be the layer potential and boundary layer potential associated to $A_{0}$. By Lemma 2.33 and Corollary 2.34, $\|f\|_{\mathrm{BMO}(\partial V)} \leq\left\|\mathcal{K}_{0} f\right\|_{\mathrm{BMO}(\partial V)}$.

We can bound $\left\|\mathcal{K}_{0} f\right\|_{\text {BMO }}$ as follows. Pick some $\Delta \subseteq \partial V$ connected. We want to bound $f_{\Delta}\left|\mathcal{K}_{0} f-c_{\Delta}\right|^{2} d \sigma$ for some constant $c_{\Delta}$. It suffices to bound this integral only for subsets $\Delta$ that satisfy $\sigma(\Delta)<\sigma(\partial V) / C$; thus, we may assume that $\Delta=\Delta\left(X_{0}, r\right)$ for some $r$ small enough that $Q=Q\left(X_{0}, r\right)$ exists. Let

$$
X_{Q}=X+(1+2 M) r \vec{e}_{j}
$$

where $\vec{e}_{j}$ is as in Definition 2.2. We have that

$$
r \approx \operatorname{dist}\left(X_{Q}, \Delta\right) \approx \operatorname{dist}\left(X_{Q}, \partial V\right) \approx \operatorname{dist}\left(X_{Q}, \partial Q\right) \approx \sigma(\Delta) \approx \sigma(\partial Q) .
$$

As shown in Section 3 of [24], since $A_{0}$ is real, if $\operatorname{div} A_{0} \nabla v=0$ in $Q$ then

$$
\int_{\partial Q}\left|N_{Q}\left(v-v\left(X_{Q}\right)\right)\right|^{2} d \sigma(X) \leq C \int_{Q}|\nabla v(X)|^{2} \operatorname{dist}(X, \partial Q) d X .
$$

Here $N_{Q}$ denotes the nontangential maximal function of formula (2.6) with $Q$ in place of $V$. Letting $v=\mathcal{D}_{0} f$, we have that

$$
\begin{aligned}
f_{\Delta}\left|\mathcal{K}_{0} f-\mathcal{D}_{0} f\left(X_{Q}\right)\right|^{2} d \sigma & \leq \frac{C}{\sigma(\Delta)} \int_{\partial Q\left(X_{0}, r\right)} N_{Q}\left(\mathcal{D}_{0} f-\mathcal{D}_{0} f\left(X_{Q}\right)\right)^{2} d \sigma \\
& \leq \frac{C}{\sigma(\Delta)} \int_{Q\left(X_{0}, r\right)}\left|\nabla \mathcal{D}_{0} f(X)\right|^{2} \operatorname{dist}(X, \partial Q) d X \\
& \leq \frac{C}{\sigma(\Delta)} \int_{B\left(X_{0}, C \sigma(\Delta)\right) \cap V}^{\left|\nabla \mathcal{D}_{0} f(X)\right|^{2} \operatorname{dist}(X, \partial V) d X}
\end{aligned}
$$

By definition of $\mathrm{BMO}(\partial V)$, this implies that

$$
\left\|\mathcal{K}_{0} f\right\|_{\mathrm{BMO}(\partial V)}^{2} \leq \sup _{X_{0} \in \partial V} \sup _{r>0} \frac{C}{r} \int_{B\left(X_{0}, r\right) \cap V}\left|\nabla \mathcal{D}_{0} f(X)\right|^{2} \operatorname{dist}(X, \partial V) d X .
$$

By the bound (3.2), if $A$ is elliptic and $t$-independent and $V$ is a two-dimensional Lipschitz domain, then

$$
\sup _{X_{0} \in \partial V} \sup _{r>0} \frac{1}{r} \int_{B\left(X_{0}, r\right) \cap V}|\nabla \mathcal{D} f(X)|^{2} \operatorname{dist}(X, \partial V) d X \leq C\|f\|_{\operatorname{BMO}(\partial V)} .
$$


The map $A \mapsto \Gamma_{X}^{A}$ is analytic in the sense that, if $z \mapsto A_{z}$ is analytic from $\mathbb{C}$ to $L^{\infty}\left(\mathbb{R}^{2} \mapsto \mathbb{C}^{2 \times 2}\right)$, then so is the map $z \mapsto \Gamma_{X}^{A_{z}}(Y)$ for any fixed $X, Y \in \mathbb{R}^{2}$. This follows from analyticity of the heat kernel (see page 57 of [7]) and the construction of the fundamental solution in [4]. From this it follows that $z \mapsto \nabla \mathcal{D}^{A_{z}} f(X)$ is analytic for any given $f \in \mathrm{BMO}$ and $X \in V$, and so

$$
\frac{1}{r} \int_{B\left(X_{0}, r\right) \cap V}\left|\nabla \mathcal{D}_{0} f(X)-\nabla \mathcal{D} f(X)\right|^{2} \operatorname{dist}(X, \partial V) d X \leq C\left\|A-A_{0}\right\|_{L^{\infty}}\|f\|_{\mathrm{BMO}}^{2} .
$$

A more detailed argument may be found in Section 4.6 of [8].

Thus by the bound (4.11),

$$
\begin{aligned}
\|f\|_{\mathrm{BMO}}^{2} \leq\left\|\mathcal{K}_{0} f\right\|_{\mathrm{BMO}}^{2} \leq & \sup _{X_{0} \in \partial V r>0} \sup _{r} \frac{C}{r} \int_{B\left(X_{0}, r\right) \cap V}|\nabla \mathcal{D} f(X)|^{2} \operatorname{dist}(X, \partial V) d X \\
& +C\left\|A-A_{0}\right\|_{L^{\infty}}\|f\|_{\mathrm{BMO}}^{2} .
\end{aligned}
$$

But since $u=\mathcal{D} f$ and $u$ satisfies the bound (4.2),

$$
\|f\|_{\mathrm{BMO}(\partial V)}^{2} \leq C \widetilde{C}^{2}+C\left\|A-A_{0}\right\|_{L^{\infty}}\|f\|_{\mathrm{BMO}(\partial V)}^{2} .
$$

Recall that we assumed $f \in \operatorname{BMO}(\partial V)$ (with no assumptions on $\|f\|_{\mathrm{BMO}(\partial V)}$ beyond $\left.\|f\|_{\mathrm{BMO}(\partial V)}<\infty\right)$. Thus the right-hand side is finite. If $\left\|A-A_{0}\right\|_{L^{\infty}}$ is small enough, we may hide the last term. Thus, $\|f\|_{\mathrm{BMO}(\partial V)} \leq C \widetilde{C}$. This completes the proof for $u$ of the form $u=\mathcal{D} f$.

We now consider more general $u$. In this case we will work as in the proof of Lemma 4.7. Again, we assume that $V$ is starlike with respect to the origin, and let $V_{\delta}=(1-\delta) V$. By Lemma 4.6, $u$ satisfies the bound (4.2) in $V_{\delta}$, uniformly in $\delta$.

But $\overline{V_{\delta}} \subset V$, so by the continuity estimate (2.13), $u$ is continuous on $\overline{V_{\delta}}$; thus $g_{\delta}=\left.u\right|_{\partial V_{\delta}} \in L^{\infty}\left(\partial V_{\delta}\right) \subset \operatorname{BMO}\left(\partial V_{\delta}\right)$. Let $\mathcal{K}_{\delta}=\mathcal{K}_{V_{\delta}}, \mathcal{D}_{\delta}=\mathcal{D}_{V_{\delta}}$, and let $N_{\delta}$ be as in formula (2.6) with $V$ replaced by $V_{\delta}$.

By Lemma 2.33 and by Corollary 2.34, there is some $\varepsilon>0$ independent of $\delta$ such that if $\|\operatorname{Im} A\|_{L^{\infty}}<\varepsilon$, then $\mathcal{K}_{\delta}$ is invertible on $\operatorname{BMO}\left(\partial V_{\delta}\right)$; we assume that $\varepsilon$ is also small enough that Lemmas 2.28 and 2.32 are valid.

Let $f_{\delta} \in \operatorname{BMO}\left(\partial V_{\delta}\right)$ be such that $\mathcal{K}_{\delta} f_{\delta}=g_{\delta}$. Since $\mathrm{BMO} \subset L^{p}$ for any $1<p<\infty$, we have that by Lemma 2.28 and Corollary $2.34, N_{\delta}\left(\mathcal{D}_{\delta} f_{\delta}\right) \in L^{p}\left(\partial V_{\delta}\right)$ for any $1<p<\infty$. Choose $p$ large enough that Lemma 2.32 is valid. Since $u \in L^{\infty}\left(\overline{V_{\delta}}\right)$, we have that $N_{\delta} u \in L^{\infty}\left(\partial V_{\delta}\right) \subset L^{p}\left(\partial V_{\delta}\right)$, and so $\mathcal{D}_{\delta} f_{\delta}=u$ in $V_{\delta}$.

So if $X_{0} \in V$, then by the remarks above,

$$
\begin{aligned}
\left(f_{\partial V_{\delta}} N_{\delta}\left(u-u\left(X_{0}\right)\right)^{p} d \sigma\right)^{1 / p} & =\left(f_{\partial V_{\delta}} N_{\delta}\left(\mathcal{D}_{\delta} f_{\delta}-\mathcal{D}_{\delta} f_{\delta}\left(X_{0}\right)\right)^{p} d \sigma\right)^{1 / p} \\
& \leq C(p) \widetilde{C} \frac{\sigma\left(\partial V_{\delta}\right)}{\operatorname{dist}\left(X_{0}, \partial V_{\delta}\right)}
\end{aligned}
$$

for any $1 \leq p<\infty$. Letting $\delta \rightarrow 0$ completes the proof. 
We may now prove Theorem 4.1.

Proof of Theorem 4.1. We wish to show that if $u$ satisfies $\operatorname{div} A \nabla u=0$ in $V$, for $\|\operatorname{Im} A\|_{L^{\infty}}$ small enough, and if $u$ satisfies the bound (4.2), then $\left.u\right|_{\partial V}$ exists and lies in $\operatorname{BMO}(\partial V)$.

Let $\Delta \subset \partial V$ be connected and small enough that $\Delta=\Delta(X, r / 2)$ for some $X$ and $r$ such that $Q=Q(X, r)$ exists. By Lemma 4.6, the bound (4.2) is valid in $Q$. Observe that $Q$ is starlike, and so we may apply Lemmas 4.7 and 4.8. By Lemma 4.8 , we have that $N_{Q}\left(u-u\left(X_{Q}\right)\right) \in L^{p}(\partial Q)$ for any $1<p<\infty$, where $X_{Q}$ is as in formula (4.10). Choosing $p$ large enough, we see that by Lemma 4.7, $u-u\left(X_{Q}\right)$ has a nontangential limit $f$ almost everywhere on $\partial Q$, and hence almost everywhere in $\Delta$.

Furthermore, by Lemma 4.8,

$f_{\Delta}\left|f-u\left(X_{Q}\right)\right|^{2} d \sigma \leq f_{\Delta} N_{Q}\left(u-u\left(X_{Q}\right)\right)^{2} d \sigma \leq C f_{\partial Q} N_{Q}\left(u-u\left(X_{Q}\right)\right)^{2} d \sigma \leq C \widetilde{C}^{2}$

and so by definition of $\operatorname{BMO}(\partial V), f \in \operatorname{BMO}(\partial V)$ with norm at most $C \widetilde{C}$.

We are left with Theorem 1.7 (the maximum principle) and Theorem 4.3 (uniqueness). We begin by proving a connection between solutions $u$ that satisfy the Carleson-measure condition (4.4) and bounded solutions.

Lemma 4.12. Let $V \subset \mathbb{R}^{2}$ be a Lipschitz domain. Suppose that $A$ is t-independent and elliptic. Then there is some $\varepsilon>0$ such that if $\|\operatorname{Im} A\|_{L^{\infty}}<\varepsilon$, then the following holds.

If $\operatorname{div} A \nabla u=0$ in $V$, if $u$ satisfies the bound (4.4), and if $f=\left.u\right|_{\partial V}$ exists and lies in $L^{\infty}(\partial V)$, then $u \in L^{\infty}(\partial V)$, with

$$
\|u\|_{L^{\infty}(V)} \leq\|f\|_{L^{\infty}(\partial V)}+C \widetilde{C}
$$

where $\widetilde{C}$ is as in formula (4.4).

Proof. Let $X \in V$. There is some $C$ large enough that, if $r=\operatorname{dist}(X, \partial V)<$ $\sigma(\partial V) / C$, then $X \in Q\left(X_{0}, 2 r\right)$ for some $X_{0} \in \partial V$, and furthermore $Q=Q\left(X_{0}, 4 r\right)$ exists. By Lemma 4.6, the bound (4.2) is valid in $Q$. Therefore, by Lemma 4.8, if $\|\operatorname{Im} A\|_{L^{\infty}}$ is small enough then

$$
\begin{aligned}
|u(X)| & \leq\left|u(X)-f_{\Delta\left(X_{0}, 4 r\right)} f d \sigma\right|+\|f\|_{L^{\infty}(\partial V)} \\
& \leq C f_{\partial Q} N_{Q}(u-u(X)) d \sigma+\|f\|_{L^{\infty}(\partial V)} \leq C \widetilde{C}+\|f\|_{L^{\infty}(\partial V)} .
\end{aligned}
$$

If $V=\Omega$ is a special Lipschitz domain this completes the proof. To deal with the case where $\partial V$ is compact and $X$ is far from $\partial V$, we next establish a pointwise bound on $\nabla u$. For any $Y \in V$, we have that by the bound (2.17),

$$
|\nabla u(Y)|^{2} \leq C f_{B(Y, \operatorname{dist}(Y, \partial V) / 2)}|\nabla u|^{2} .
$$


Let $Y^{*} \in \partial V$ with $r=\operatorname{dist}(Y, \partial V)=\left|Y-Y^{*}\right|$. By the bound (4.4),

$$
\begin{aligned}
|\nabla u(Y)|^{2} & \leq C f_{B(Y, r / 2)}|\nabla u(Z)|^{2} \frac{\operatorname{dist}(Z, \partial V)}{r} d Z \\
& \leq \frac{C}{r^{3}} \int_{V \cap B\left(Y^{*}, 2 r\right)}|\nabla u(Z)|^{2} \operatorname{dist}(Z, \partial V) d Z \leq \frac{C}{r^{3}} \sigma(\partial V) \widetilde{C}^{2} .
\end{aligned}
$$

Now, let $X \in V$ with $\operatorname{dist}(X, \partial V)>\sigma(\partial V) / C$. Let $X_{1} \in V$ satisfy $\operatorname{dist}\left(X_{1}, \partial V\right)=$ $\sigma(\partial V) / C$, and let $\omega$ be a path from $X_{1}$ to $X$. Then

$$
\begin{aligned}
|u(X)| & \leq\left|u(X)-u\left(X_{1}\right)\right|+\left|u\left(X_{1}\right)\right| \leq \int_{\omega}|\nabla u(Y)| d \sigma(Y)+C \widetilde{C}+\|f\|_{L^{\infty}(\partial V)} \\
& \leq C \widetilde{C} \sqrt{\sigma(\partial V)} \int_{\omega} \frac{1}{\operatorname{dist}(Y, \partial V)^{3 / 2}} d \sigma(Y)+C \widetilde{C}+\|f\|_{L^{\infty}(\partial V)} .
\end{aligned}
$$

An appropriate choice of $X_{1}$ and $\omega$ yields that the integral is at most $C / \sqrt{\sigma(\partial V)}$, and the lemma is proven.

By Lemma 4.12 and Theorem 3.1, if $f \in L^{\infty}(\partial V) \subset \operatorname{BMO}(\partial V)$ for a Lipschitz domain $V$ with connected boundary, then (for appropriate $A$ ) there is a solution $u$ to the Dirichlet problem (1.1) that satisfies $\|u\|_{L^{\infty}(V)} \leq C\|f\|_{L^{\infty}(\partial V)}$. This proves the existence half of Theorem 1.7.

We will conclude this paper by proving uniqueness of bounded solutions. This will immediately complete the proof of Theorem 1.7. If $\left.u\right|_{\partial V}$ is a constant and $u$ satisfies the bound (4.4), then by Lemma $4.12 u \in L^{\infty}(V)$, and so Lemma 4.13 will imply that $u$ is a constant; this proves Theorem 4.3 , completing the proof of Theorem 1.4.

Lemma 4.13. Let $V \subset \mathbb{R}^{2}$ be a Lipschitz domain and let $A$ be elliptic and $t$ independent. Then there is some $\varepsilon>0$ such that, if $\|\operatorname{Im} A\|_{L^{\infty}}<\varepsilon$, if $\operatorname{div} A \nabla u=0$ in $V$ with $\left.u\right|_{\partial V}=0$, and if $u \in L^{\infty}(V)$, then $u \equiv 0$ in $V$.

Proof. We first show that $\nabla u \in L^{2}(V)$; we will then use the Caccioppoli inequality to show that $u$ is constant in $V$.

For the sake of simplicity assume that $\|u\|_{L^{\infty}(V)} \leq 1$. Consider $Q=Q\left(X_{0}, r\right)$ for some $X_{0} \in \partial V$ and some $r>0$ small enough that $Q\left(X_{0}, r\right)$ exists. Let $f=u$ on $\partial Q$. Choose some $\delta>0$, and let $g_{\delta}=f=0$ on $\partial V \cap \partial Q$, and $g_{\delta}=f=u$ on $\omega_{\delta}=\{X \in \partial Q: \operatorname{dist}(X, \partial V)>\delta\}$. By Lemma 2.9 and the bound (2.17), $|\nabla u| \leq C / \delta$ on $\omega_{\delta}$; we may choose $g_{\delta}$ such that $\left|\partial_{\tau} g_{\delta}\right|<C / \delta$ on all of $\partial Q$, where $\partial_{\tau} g_{\delta}$ is the tangential derivative of $g_{\delta}$ along $\partial Q$.

Let $v_{\delta}$ solve the Dirichlet problem (2.31) in $Q$ with boundary data $g_{\delta}$. By Lemma 2.32 and preceding remarks, if $\|\operatorname{Im} A\|_{L^{\infty}}$ is small enough then there is some $p$, with $1<p<\infty$, such that $v$ exists, and such that

$$
\left\|N_{Q}\left(u-v_{\delta}\right)\right\|_{L^{p}(\partial Q)} \leq C\left\|f-g_{\delta}\right\|_{L^{p}(\partial Q)} \leq C \delta^{1 / p}
$$

We will show that $\nabla v_{\delta} \in L^{2}(Q)$, and then use this fact to prove estimates on $u$. 
Observe that if $1 \leq q \leq \infty$, then $\partial_{\tau} v_{\delta} \in L^{q}(\partial Q)$ (with, however, a norm depending on $\delta$ ). This means that $v_{\delta}$ is a solution to the so-called Dirichlet regularity problem; this problem was investigated in [8] along with the $L^{p}$-Dirichlet problem. We will need the fact that $\nabla v_{\delta} \in L^{2}(Q)$ (possibly with a norm depending on $\delta$ ); this fact follows from Theorems 8.5, 1.6 and Lemma 3.3 in [8]. To eliminate the dependence on $\delta$, we apply Remark 2.10 to see that

$$
\int_{Q\left(X_{0}, r / 2\right)}\left|\nabla v_{\delta}\right|^{2} \leq \frac{C}{r^{2}} \int_{Q\left(X_{0}, r\right)}\left|v_{\delta}\right|^{2}
$$

But $\left\|N_{Q} v_{\delta}\right\|_{L^{p}(\partial Q)} \leq C r^{1 / p}$; an elementary argument (see Lemma 3.3 in [8]) yields that $\left\|v_{\delta}\right\|_{L^{2}(Q)} \leq C\left\|N_{Q} v_{\delta}\right\|_{L^{1}(\partial Q)}$, and so $\left\|\nabla v_{\delta}\right\|_{L^{2}\left(Q\left(X_{0}, r / 2\right)\right)}$ is bounded, uniformly in $\delta$.

Let $Q_{\varepsilon}=\left\{X \in Q\left(X_{0}, r / 2\right): \operatorname{dist}(X, \partial Q)>\varepsilon\right\}$. By Lemma 2.9, we have that

$$
\left\|\nabla u-\nabla v_{\delta}\right\|_{L^{2}\left(Q_{\varepsilon}\right)} \leq \delta^{1 / p} \frac{C r}{\varepsilon} .
$$

By choosing $\delta$ small enough, we see that $\nabla u \in L^{2}\left(Q_{\varepsilon}\right)$, uniformly in $\varepsilon$; thus $\nabla u \in L^{2}\left(Q\left(X_{0}, r / 2\right)\right)$. If $V$ is bounded, then by covering a neighborhood of $\partial V$ with such tents and by applying the definition of weak solution (specifically, the assumption that $\left.\nabla u \in L_{l o c}^{2}(V)\right)$, we see that $\nabla u \in L^{2}(V)$. Then Remark 2.10 immediately implies that $\nabla u \equiv 0$ in $V$. that

Otherwise, $\nabla u \in L^{2}(V \cap B(0, r))$ for any $r>0$. Applying Remark 2.10, we see

$$
\int_{V \cap B(0, r)}|\nabla u|^{2} \leq \frac{C}{r^{2}} \int_{V \cap B(0,2 r)}|u|^{2} \leq C,
$$

and so, letting $r \rightarrow \infty$, we see that $\nabla u \in L^{2}(V)$.

Now, fix some $r>0$, and let $\varphi$ be smooth, supported in $B(0,2 r)$ and identically equal to 1 in $B(0, r)$, with $|\nabla \varphi| \leq C / r$. As in the proof of the Caccioppoli inequality,

$$
\lambda \int_{B(0, r) \cap V}|\nabla u|^{2} \leq \operatorname{Re} \int_{V} \varphi^{2} \nabla \bar{u} \cdot A \nabla u=-\operatorname{Re} \int_{V} 2 \bar{u} \varphi \nabla \varphi \cdot A \nabla u,
$$

and so, by Hölder's inequality,

$$
\int_{B(0, r) \cap V}|\nabla u|^{2} \leq \frac{C}{r} \int_{V \cap B(0,2 r) \backslash B(0, r)}|u||\nabla u| \leq C\|u\|_{L^{\infty}}\left(\int_{V \cap B(0,2 r) \backslash B(0, r)}|\nabla u|^{2}\right)^{1 / 2} .
$$

But since $\nabla u \in L^{2}(V)$, the right-hand side must go to zero as $r \rightarrow \infty$; thus $\nabla u \equiv 0$ in $V$ and so $u$ is identically equal to zero.

\section{References}

[1] Alfonseca, M., Auscher, P., Axelsson, A., Hofmann, S. and Kim, S.: Analyticity of layer potentials and $L^{2}$ solvability of boundary value problems for divergence form elliptic equations with complex $L^{\infty}$ coefficients. Adv. Math. 226 (2011), no. 5, 4533-4606. 
[2] Auscher, P., Axelsson, A. And Hofmann, S.: Functional calculus of Dirac operators and complex perturbations of Neumann and Dirichlet problems. J. Funct. Anal. 255 (2008), no. 2, 374-448.

[3] Auscher, P., Axelsson, A. And McIntosh, A.: Solvability of elliptic systems with square integrable boundary data. Ark. Mat. 48 (2010), no. 2, 253-287.

[4] Auscher, P., Mcintosh, A. And Tchamitchian, P.: Heat kernels of second order complex elliptic operators and applications. J. Funct. Anal. 152 (1998), no. 1, 22-73.

[5] Auscher, P. And Mourgoglou, M.: Boundary layers, Rellich estimates and extrapolation of solvability for elliptic systems.. Proc. Lond. Math. Soc. (3) 109 (2014), no. $2,446-482$.

[6] Auscher, P. And Tchamitchinan, P.: Calcul fontionnel précisé pour des opérateurs elliptiques complexes en dimension un (et applications à certaines équations elliptiques complexes en dimension deux). Ann. Inst. Fourier (Grenoble) 45 (1995), no. $3,721-778$.

[7] Auscher, P. And Tchamitchian, P.: Square root problem for divergence operators and related topics. Astérisque, 249, 1998.

[8] Barton, A.: Elliptic partial differential equations with almost-real coefficients. Mem. Amer. Math. Soc. 223 (2013), no. 1051, vii+108.

[9] DahlBerg, B.: Weighted norm inequalities for the Lusin area integral and the nontangential maximal functions for functions harmonic in a Lipschitz domain. Studia Math. 67 (1980), no. 3, 297-314.

[10] Dahlberg, B., Jerison, D. and Kenig, C.: Area integral estimates for elliptic differential operators with nonsmooth coefficients. Ark. Mat. 22 (1984), no. 1, 97-108.

[11] De Giorgi, E.: Sulla differenziabilità e l'analiticità delle estremali degli integrali multipli regolari. Mem. Accad. Sci. Torino. Cl. Sci. Fis. Mat. Nat. (3) 3 (1957), $25-43$.

[12] Dindos, M., Kenig, C. And Pipher, J.: BMO solvability and the $A_{\infty}$ condition for elliptic operators. J. Geom. Anal. 21 (2011), no. 1, 78-95.

[13] Fabes, E., Johnson, R. And Neri, U.: Spaces of harmonic functions representable by Poisson integrals of functions in BMO and $\mathcal{L}_{p, \lambda}$. Indiana Univ. Math. J. 25 (1976), no. 2, 159-170.

[14] Fabes, E. And Neri, U.: Dirichlet problem in Lipschitz domains with BMO data. Proc. Amer. Math. Soc. 78 (1980), no. 1, 33-39.

[15] Fefferman, C.: Characterizations of bounded mean oscillation. Bull. Amer. Math. Soc. 77 (1971), 587-588.

[16] Fefferman, C. And Stein, E.: $H^{p}$ spaces of several variables. Acta Math. 129 (1972), no. 3-4, 137-193.

[17] Frehse, J.: An irregular complex valued solution to a scalar uniformly elliptic equation. Calc. Var. Partial Differential Equations 33 (2008), no. 3, 263-266.

[18] Grau de la Herran, A. and Hofmann, S.: Generalized local Tb theorems for square functions, and applications. ArXiv: 1212.5870v1, 2012.

[19] Hofmann, S.: Local $T(b)$ theorems and applications in PDE. In Harmonic analysis and partial differential equations, 29-52. Contemp. Math. 505, Amer. Math. Soc., Providence, RI, 2010.

[20] Hofmann, S., Kenig, C., Mayboroda, S. And Pipher, J.: Square function/nontangential maximal function estimates and the Dirichlet problem for non-symmetric elliptic operators. J. Amer. Math. Soc. 28 (2015), no. 2, 483-529. 
[21] Hofmann, S., Kenig, C., Mayboroda, S. And Pipher, J.: The regularity problem for second order elliptic operators with complex-valued bounded measurable coefficients. Math. Ann. 361 (2015), no. 3-4, 863-907.

[22] Hofmann, S. And Kim, S.: The Green function estimates for strongly elliptic systems of second order. Manuscripta Math. 124 (2007), no. 2, 139-172.

[23] Jerison, D. And Kenig, C.: The Dirichlet problem in nonsmooth domains. Ann. of Math. (2) 113 (1981), no. 2, 367-382.

[24] Kenig, C., Koch, H., Pipher, J. And Toro, T.: A new approach to absolute continuity of elliptic measure, with applications to non-symmetric equations. Adv. Math. 153 (2000), no. 2, 231-298.

[25] Kenig, C. And Ni, W. M.: On the elliptic equation $L u-k+K \exp [2 u]=0$. Ann. Scuola Norm. Sup. Pisa Cl. Sci. (4) 12 (1985), no. 2, 191-224.

[26] Kenig, C. And Pipher, J.: The Neumann problem for elliptic equations with nonsmooth coefficients. Invent. Math. 113 (1993), no. 3, 447-509.

[27] Kenig, C. And Rule, D.: The regularity and Neumann problem for non-symmetric elliptic operators. Trans. Amer. Math. Soc. 361 (2009), no. 1, 125-160.

[28] Mazja, W., Nasarow, S. and Plamenewski, B.: Asymptotische Theorie elliptischer Randwertaufgaben in singulär gestörten Gebieten. I. Störungen isolierter Randsingularitäten. Mathematische Lehrbücher und Monographien II. Abteilung: Mathematische Monographien 82, Akademie-Verlag, Berlin 1991.

[29] Meyers, N.: An $L^{p}$-estimate for the gradient of solutions of second order elliptic divergence equations. Ann. Scuola Norm. Sup. Pisa (3) 17 (1963), 189-206.

[30] Morrey, JR., C.: Multiple integrals in the calculus of variations.. Die Grundlehren der mathematischen Wissenschaften 130, Springer-Verlag, New York, 1966.

[31] Moser, J.: On Harnack's theorem for elliptic differential equations. Comm. Pure Appl. Math. 14 (1961), 577-591.

[32] Nash, J.: Continuity of solutions of parabolic and elliptic equations. Amer. J. Math. 80 (1958), 931-954.

[33] Rosén, A.: Layer potentials beyond singular integral operators. Publ. Mat. 57 (2013), no. 2, 429-454.

[34] Rule, D.: Non-symmetric elliptic operators on bounded Lipschitz domains in the plane. Electron. J. Differential Equations (2007), no. 144, 1-8.

[35] Semmes, S.: Square function estimates and the T(b) theorem. Proc. Amer. Math. Soc. 110 (1990), no. 3, 721-726.

[36] Stein, E. M.: Harmonic analysis: real-variable methods, orthogonality, and oscillatory integrals. Princeton Mathematical Series 43, Monographs in Harmonic Analysis III, Princeton Univ. Press, Princeton, NJ, 1993.

Received September 26, 2013.

Ariel Barton: 202 Math Sciences Bldg., University of Missouri, Columbia, MO 65211, USA.

E-mail: bartonae@missouri.edu 